\title{
Relativistic scattering of a fast spinning neutron star by a massive black hole
}

\author{
Kaye Jiale $\mathrm{Li}^{1,2 \star}$, Kinwah $\mathrm{Wu}^{1}$, Po Kin Leung ${ }^{2}$ and Dinesh Singh ${ }^{3}$ \\ ${ }^{1}$ Mullard Space Science Laboratory, University College London, Holmbury St Mary, Surrey, RH5 6NT, United Kingdom \\ ${ }^{2}$ Department of Physics, Chinese University of Hong Kong, Shatin, NT, Hong Kong SAR, China \\ ${ }^{3}$ Department of Physics, University of Regina, Regina, SK S4S 0A2, Canada
}

Accepted 2021 October 5. Received 2021 October 4; in original form 2021 February 3

\begin{abstract}
The orbital dynamics of fast spinning neutron stars encountering a massive Black Hole (BH) with unbounded orbits are investigated using the quadratic-in-spin Mathisson-Papapetrou-Dixon (MPD) formulation. We consider the motion of the spinning neutron stars with astrophysically releyant speed in the gravity field of the BH. For such slow-speed scattering, the hyperbolic orbits followed by these neutron stars all have near the $e=1$ eccentricity, and have distinct properties compared with those of $e \gg 1$. We have found that compared with geodesic motion, the spin-orbit and spinspin coupling will lead to a variation of scattering angles at spatial infinity, and this variation is more prominent for slow-speed scattering than fast-speed scattering. Such a variation leads to an observable difference in pulse-arrival-time within a few hours of observation, and up to a few days or months for larger BH masses or longer spinning periods. Such a relativistic pulsar-BH system also emits a burst of gravitational waves (GWs) in the sensitivity band of LISA, and for optimal settings, can be seen up to $100 \mathrm{Mpc}$ away. A radio follow up of such a GW burst with SKA or FAST will allow for measuring the orbital parameters with high accuracy and testing the predictions of General Relativity (GR).
\end{abstract}

Key words: black hole physics - gravitation - celestial mechanics - relativistic processes - pulsars general

\section{INTRODUCTION}

Pulsars are either fast spinning young neutron stars with a strong magnetic field $\left(\sim 10^{12}-10^{13} \mathrm{G}\right)$ or old recycled neutron stars with a weaker magnetic field $\left(\sim 10^{9} \mathrm{G}\right)$ (see e.g. Lorimer 2008). The latter are millisecond pulsars (MSPs), whose spin periods range from $\sim 1-10 \mathrm{~ms}$ and are extremely stable, with a drift of roughly 1 pulse period on a Hubble timescale (see e.g. Manchester 2017). MSPs are mass gyros, and the stability of their spin periods make them very high-precision timing instruments. Relativistic binaries containing an MSP orbiting around a BH, i.e. MSP - BH binaries, are particularly useful for the investigation of fundamental physics, where accurate, reliable measurements are essential. The two subclasses of the MSP - BH binaries, the extreme-mass-ratio binary (EMRB) and the extreme-massratio-inspiral (EMRI) systems, each contain a massive $\mathrm{BH}$. These systems are natural GW sources, expected to be detected by the Laser Interferometric Space Antenna (LISA) (see Amaro-Seoane et al. 2007; Gair et al. 2017). As MSPs are radio sources, an "observable" electromagnetic counter-

^ E-mail: j-li.19@ucl.ac.uk (KJL), kinwah.wu@ucl.ac.uk (KW), pkleung@phy.cuhk.edu.hk (PKL), dinesh.singh@uregina.ca (DS) part will be present if an EMRB/EMRI GW event occurs, allowing high-precision measurements to be made in the electromagnetic messenger domain and in the gravitational messenger domain independently. In the theoretical perspective, the fact that neutron stars have a relatively narrow mass range (see Lattimer 2012; Özel \& Freire 2016) reduces one important system parameter in the orbital and spin dynamical modelling. Moreover, as the mass of a neutron star is small in comparison with the massive nuclear BH, the MSP can be treated as a test object.

In the test-object limit, the motion of a spinning secondary in the gravity field of a massive black hole is governed by the MPD equations. Most of the current studies (e.g. Semerák 1999; Yunes et al. 2011; Singh et al. 2014; Kimpson et al. 2020a,b) using MPD equations have put focus on bounded systems and their orbital dynamics. Also a circular or a quasi-circular orbit approximation is often adopted (e.g. Bini et al. 2005; Han 2010; Velandia \& Tejeiro 2018; Chen et al. 2019), where the spin-spin and spin-orbit couplings are treated as independent separated components (see also the application of same deposition in the study of binary neutron stars around a black hole in Remmen \& Wu 2013). Compared to the bounded orbits, hyperbolic orbits admit the advantage of having clean non-degenerate 
observables. Among them the most well recognised is the scattering angle $\chi$ ( $\equiv \Delta \phi$ in this paper). The correction to the scattering angle due to the spin of the secondary has been widely studied. For example, Bini \& Geralico (2017); Bini, Geralico \& Vines (2017) calculated the analytical formula (to the first order in spin) of the orbit of a spinning particle on the equatorial plane around a massive $\mathrm{BH}$ using the MPD equations. Bini \& Geralico (2018) studied the effects of spin and spin-induced quadrupolar moments on the scattering angle in the high-energy limit. MPD equations are also adopted in the theoretical modelling of scattering system by Vines, Kunst, Steinhoff \& Hinderer (2016); Vines (2018); Vines, Steinhoff \& Buonanno (2019); Antonelli, Kavanagh, Khalil, Steinhoff \& Vines (2020), etc. While analytical analysis of the dynamics of spinning binary tend to restrict the orbits to equatorial plane, where the spin is aligned or anti-aligned with the orbital angular momentum, the orbits can be complicated and even chaotic (see e.g. Suzuki \& Maeda 1997; Hartl 2003a,b; Kao \& Cho 2005; Zelenka et al. 2019; Witzany et al. 2019; Zelenka et al. 2020) when the spin of the secondary is not aligned with the orbital angular momentum. The rich dynamical features of non-equatorial motions are studied by Singh, Wu \& Sarty (2014); Han \& Cheng (2017); Witzany (2019); Li, Wu \& Singh (2019); Kimpson, Wu \& Zane (2019); Keresztes \& Mikóczi (2019); Keresztes \& Mikoczi (2020), etc. There are also a few studies (e.g. Hansen 1972; Majár et al. 2010; De Vittori et al. 2014) focusing on the gravitational radiation from unbounded systems.

Here we investigate in the astrophysical context the dynamics of the unbounded EMRB, where an MSP (i.e., spinning mass gyro with a radio beaming beacon) interacts with a stationary space-time provided by a massive BH. The focus of the work is on the non-linearity and complexity arisen from the spin-couplings in a fly-by encounter, i.e. the MSP is in an unbounded orbit with respect to the BH. The MPD formulation is adopted to derive the orbital and spin evolutionary equations of the MSP. By solving the orbital and spin evolutionary equation we determine the multi-messenger signatures of these system. We organise the paper as follows. In $\S 2$ we present the MPD equations of motion (EOMs) for the astrophysically relevant parameter space that concerns this paper. In $\S 3$ we extend the numericalsolution to radial infinity to compare the scattering angles with the linearin-spin analytical formula from Bini \& Geralico (2017) and Bini et al. (2017), show the complex orbital dynamics of the scattering, and investigate the detectability of the spin's effects in such an EMRB system; In $\$ 4$ we comment on the existence of such EMRB systems and the implications in astrophysics and multi-méssenger observations, after which we conclude the paper in $\S 5$.

\section{UNBOUNDED SCATTERING BETWEEN NEUTRON STARS AND A MASSIVE BH}

We adopt a $[-+++]$ metric signature and a natural unit system with unity speed of light $c$ and gravitational constant $G$ (i.e. $c=G=1$ ). The Schwarzschild radius of a $\mathrm{BH}$ is therefore $r_{\mathrm{s}}=2 M$. The pulsar is a spinning test object, and its mass is fixed to be $1.5 \mathrm{M}_{\odot}$, a value typical for a neutron star. The dimensionless spin of the neutron star with period
$P_{\mathrm{ns}}$ is set to be

$\hat{s}=\frac{s}{m M}=\left(\frac{1 \mathrm{~ms}}{P_{\mathrm{ns}}}\right)\left(\frac{10^{3} \mathrm{M}_{\odot}}{M}\right) \times 5.68 \times 10^{-4}$,

under the assumption that the pulsar is a solid sphere with uniform density and radius $10 \mathrm{~km}$. We refer to $P_{\mathrm{ns}}=1 \mathrm{~ms}$ pulsars as MSPs.

We adopt the MPD formulation up to quadrupole interaction. The orbital and spin evolutions of the pulsar are governed by the equations:

$$
\begin{aligned}
\dot{p}^{\mu} & =-\frac{1}{2} R^{\mu}{ }_{\nu \alpha \beta} u^{v}{ }_{s}^{\alpha \beta}+\mathcal{F}^{\mu} ; \\
\dot{s}^{\mu \nu} & =p^{\mu} u^{v}-p^{v} u^{\mu}+\mathcal{T}^{\mu \nu} \\
\mathcal{F}^{\mu} & \equiv-\frac{1}{6} J^{\alpha \beta \gamma \sigma} \nabla^{\mu} R_{\alpha \beta \gamma \sigma}, \\
\mathcal{T}^{\mu \nu} & \equiv \frac{4}{3} J^{\alpha \beta \gamma[\mu} R^{\nu]}{ }_{\gamma \alpha \beta} .
\end{aligned}
$$

(see Mathisson 1937; Papapetrou 1951; Dixon 1964), where $u^{\mu}=\mathrm{d} x^{\mu} / \mathrm{d} \tau$ is the unit tangent vector along the worldline of the pulsar's centre of mass. The over dot represents the covariant derivative along this worldline, i.e. $\dot{p}^{\mu} \equiv u^{\nu} \nabla_{v} p^{\mu}$. The Dixon force and torque are $\mathcal{F}^{\mu}$ and $\mathcal{T}^{\mu \nu}$, respectively, and $J^{\alpha \beta \gamma \sigma}$ is the quadrupole tensor of the neutron star. We adopt the spin-induced quadrupole tensor as used in Steinhoff (2011).

$$
\begin{aligned}
& J^{\alpha \beta \gamma \sigma}=4 v^{[\alpha} \chi(v)^{\beta][\gamma} v^{\sigma]}, \text { with } \\
& \chi(v)^{\beta \gamma}=\frac{3}{4} \frac{C Q}{m}\left[s^{\beta} s^{\gamma}-\frac{1}{3} s^{2}\left(g^{\beta \gamma}+v^{\beta} v^{\gamma}\right)\right] .
\end{aligned}
$$

and $v^{\alpha} \equiv p^{\alpha} / m$, where $m \equiv \sqrt{-p^{\mu} p_{\mu}}$. $C_{Q}$ is the polarizability constant and its value depends on the equation of state of the object. It is normalised such that $C_{Q}=1$ corresponds to a $\mathrm{BH}$. For neutron star, the value of $C_{Q}$ is chosen to be between 3.1 and 7.4 (Laarakkers \& Poisson 1999) ${ }^{1}$. Urbanec et al. (2013) found a value between 5-6. We take $C_{Q}$ to be 6 and argue that the main findings in this work are dominated by spin-couplings. The covariant derivatives are taken with respect to a background metric, which is provided by a Kerr $\mathrm{BH}$, with the line element given by

$$
\begin{aligned}
-\mathrm{d} \tau^{2}= & -\left(1-\frac{2 M r}{\Sigma}\right) \mathrm{d} t^{2}-\frac{4 a M r \sin ^{2} \theta}{\Sigma} \mathrm{d} t \mathrm{~d} \phi \\
& +\frac{\Sigma}{\Delta} \mathrm{d} r^{2}+\Sigma \mathrm{d} \theta^{2}+\left(r^{2}+a^{2}+\frac{2 a^{2} M r \sin ^{2} \theta}{\Sigma}\right) \\
& \times \sin ^{2} \theta \mathrm{d} \phi^{2},
\end{aligned}
$$

in Boyer-Lindquist coordinates. Here $\Sigma=r^{2}+a^{2} \cos ^{2} \theta$, $\Delta=r^{2}-2 M r+a^{2}$ and $(r, \theta, \phi)$ represent the spatial 3-vector in (pseudo-)spherical polar coordinates with the black-hole centre as the origin.

Here we consider the Tulczyjew-Dixon (TD) spin supplementary condition (see Tulczyjew 1959; Deriglazov \& Ramírez 2017), i.e.

$s^{\mu v} p_{v}=0$,

1 Notice that they did not study $1.5 \mathrm{M}_{\odot}$ but $1.4 \mathrm{M}_{\odot}$ and $1.6 \mathrm{M}_{\odot}$ neutron stars. 
and use the spin-vector to simplify the EOMs:

$s_{\mu}=-\frac{1}{2 m} \epsilon_{\mu \nu \alpha \beta} p^{v} s^{\alpha \beta} ;$

$s^{\mu \nu}=\frac{1}{m} \epsilon^{\mu \nu \alpha \beta} p_{\alpha} s_{\beta}$,

with Levi-Civita tensor $\epsilon_{\mu \nu \alpha \beta}=\sqrt{-g} \sigma_{\mu \nu \alpha \beta}$ adopting the $\sigma_{0123}=+1$ permutation.

Under this spin supplementary condition, the EOMs now become

$$
\begin{aligned}
\frac{\mathrm{d} p^{\alpha}}{\mathrm{d} \tau}= & -\Gamma_{\mu \nu}^{\alpha} p^{\mu} u^{\nu}+\lambda\left(-\frac{1}{2} R_{\beta \mu \nu}^{\alpha} u^{\beta} s^{\mu \nu}+\mathcal{F}^{\alpha}\right) \\
\frac{\mathrm{d} s^{\alpha}}{\mathrm{d} \tau}= & -\Gamma_{\mu \nu}^{\alpha} s^{\mu} u^{\nu}+\lambda\left[\left(-\frac{1}{2 m^{2}} R_{\gamma \beta \mu \nu} u^{\beta} s^{\mu \nu}+\mathcal{F}_{\gamma}\right) s^{\gamma} p^{\alpha}\right. \\
& \left.-\frac{1}{2 m} \epsilon_{\beta \mu \nu}^{\alpha} p^{\beta} \mathcal{T}^{\mu \nu}\right]
\end{aligned}
$$

where we follow the method in Singh (2005) and Singh et al. (2014) to introduce the parameter switch $\lambda$ into the MPD equations. The spin-curvature coupling is included when setting $\lambda=1$. Further, quadrupole-curvature coupling is included when both $C_{Q}$ and $\lambda$ are non-zero. With these two switches, we can compare the difference in the dynamics between the presence and the absence of spin-curvature and quadrupole-curvature couplings.

It remains to determine the 4-velocity. We follow the procedure of Semerák (1999); Han \& Cheng (2017) to first contract Eq. 3 with $p_{v}$, making use of the relation $R_{v \sigma \alpha \beta} s^{\mu \nu}{ }^{\sigma \beta}=-R_{v \sigma \alpha \beta} s^{v \sigma}{ }^{\mu \beta} / 2$, and then making use of the TD spin supplementary condition to replace $p_{\mu} \dot{s}^{\nu \mu}$ with $\dot{p}_{\mu} s^{\mu \nu}$. Finally, the 4-velocity takes the following form:

$$
\begin{aligned}
\frac{\mathrm{d} x^{\alpha}}{\mathrm{d} \tau}= & u^{\alpha}=\frac{m_{u}}{m^{2}}\left(p^{\alpha}-\dot{p}_{\nu} s^{\alpha \nu}\right)-\frac{1}{m^{2}} p_{\nu} F^{\alpha \nu}, \\
\dot{p}_{\mu} s^{\eta \mu}= & \frac{1}{4 m^{2}+R_{\mu \nu \alpha \beta} s^{\alpha \beta} s^{\mu \nu}}\left(-2 m_{u} R_{\mu \nu \alpha \beta} s^{\alpha \beta} p^{v} s^{\eta \mu}\right. \\
& \left.+2 R_{\mu \nu \alpha \beta} s^{\alpha \beta} p_{\delta} \mathcal{T}^{\nu} s^{\eta \mu}-4 m^{2} \mathcal{F}_{\mu} s^{\eta \mu}\right),
\end{aligned}
$$

where $m_{u} \equiv-p^{\mu} u_{\mu}$ is the mass measured by an observer with 4 -velocity $u^{\mu}$. These two masses $m$ and $m_{u}$ are, in general, not the same. As a set of non-linear equations, (12) is underdetermined, but a unique solution can be obtained by use of the normalisation condition $u_{\mu} u^{\mu}=-1$. The MPD formula does not require $u^{\mu}$ to be unit-normal. For example, Ehlers \& Rudolph (1977) assumed $u_{\mu} p^{\mu}=-m$ (they used $\dot{z}^{\mu}$ instead of $u_{\mu}$ ) by scaling the time parameter of the curve. Therefore, requiring $u^{\mu}$ to be either unit-normal or to satisfy $u_{\mu} p^{\mu}=-m$ are two distinct choices. In this study we have chosen $u^{\mu}$ to be unit-normal, such that the time parameter becomes the proper time.

There are several constants of motion related to the MPD equation under TD spin supplementary condition. The Killing vector field $\xi^{\mu}=(1,0,0,0)$ and $\eta^{\mu}=(0,0,0,1)$ give rise to the conservation of energy and angular momentum:

$$
\begin{gathered}
E=-\xi_{\alpha} p^{\alpha}+\frac{1}{2} s^{\alpha \beta} \nabla_{\beta} \xi_{\alpha}, \\
J=\eta_{\alpha} p^{\alpha}-\frac{1}{2} s^{\alpha \beta} \nabla_{\beta} \eta_{\alpha},
\end{gathered}
$$

where $\nabla_{\beta} \xi_{\alpha}=g_{t[\alpha, \beta]}$ and $\nabla_{\beta} \eta_{\alpha}=g_{\phi[\alpha, \beta]}$. There is a further constant of motion that is conserved at first order in spin given by the Killing-Yano tensor (Rudiger 1981, 1983; Witzany 2019):

$$
K=Y_{\mu \chi} Y_{v}{ }^{\chi} p^{\mu} p^{\nu}-2 \lambda p^{\mu} s^{\rho \sigma}\left(Y_{\mu \rho ; \kappa} Y^{\kappa}{ }_{\sigma}+Y_{\rho \sigma ; K} Y_{\mu}^{K}\right),
$$

where $Y_{\mu \nu}$ is the Killing-Yano tensor with components listed in Eq. 2 of Witzany (2019). The dynamical mass $m$ is a constant of motion at dipole order, but varies as $O\left(s^{2}\right)$ at the quadrupole order. A mass-like definition

$m_{J} \equiv m-\frac{1}{6} R_{\alpha \beta \mu v} J^{\alpha \beta \mu \nu}$,

(Steinhoff 2011) varies as $O\left(s^{4}\right)$ at the quadrupole order. Although the magnitude of spin

$$
s^{2} \equiv \frac{1}{2} s^{\mu v} s_{\mu v} \equiv s^{\mu} s_{\mu}
$$

is not conserved for a general quadrupole tensor, it is conserved for this ansatz spin-induced quadrupole tensor.

The initial conditions are set to be $x^{\mu}=\left(0,10^{4} M, \pi / 2,0\right)$, corresponding to a pulsar on $x$-axis, at $t=\tau=\theta$. At such large radial distance, we have $p^{\mu}=m u^{\mu}$ with $m=1.5 \mathrm{M}_{\odot}$, and therefore $p_{t} \equiv-m E_{0}$ and $p_{\phi} \equiv m J_{0}$, where $E_{0}$ and $J_{0}$ are defined in Eq. A2. Further, the test object is initially moving on the equatorial plane and we have $p^{\theta}=0$. Then, $p^{r}$ can be determined by the condition $p^{\mu} p_{\mu}=-m^{2}$. The initial values of the 4 -spin are determined by $\theta_{\mathrm{S}}$ and $\phi_{\mathrm{S}}$ which are defined with repect to a Cartesian coordinate in the MSP's local tetrad frame, which is approximately equivalent to the global Cartesian coordinate at large radial distance. For example, when $\theta_{\mathrm{S}}=90^{\circ}, \phi_{\mathrm{S}}=0^{\circ}$, the 3 -spin is in radial direction, and when $\theta_{S}=0^{\circ}$, the 3 -spin is parallel to the angular momentum.

\subsection{Hyperbolic orbits of MSPs around BH}

For a massive nuclear $\mathrm{BH}$, stellar interactions dominate outside the influence radius of the $\mathrm{BH}$ :

$r_{\text {inf }} \equiv \frac{G M}{\sigma^{2}}$,

where $\sigma$ is the velocity dispersion. If intermediate-mass BHs do follow the M- $\sigma$ relation, then $\sigma \approx 18 \mathrm{~km} / \mathrm{s}$ for an $M=10^{3} \mathrm{M}_{\odot} \mathrm{BH}$ (McConnell et al. 2011), giving rise to an influence radius of about $0.01 \mathrm{pc}$, while $M=10^{5} \mathrm{M}_{\odot}$ BHs have an influence radius of about $0.2 \mathrm{pc}$. Measurements of globular clusters (GCs) give similar results, ranging from $3 \mathrm{~km} / \mathrm{s}$ to $20 \mathrm{~km} / \mathrm{s}$ (Baumgardt \& Hilker 2018).

If a star is scattered into a hyperbolic orbit around the $\mathrm{BH}$, the star's orbit and periapsis distance are uniquely described by its initial velocity (velocity at $r \rightarrow \infty$, we denote $v_{\infty}$ ) and impact parameter $b$ (or angular momentum $L_{z}$ ). Stars with smaller initial velocities can get closer to the $\mathrm{BH}$ even when the impact parameter is large, because of their small inertia. The impact parameter is related to the angular momentum $J_{0}$ and $v_{\infty}$ via:

$$
\begin{aligned}
b & \equiv \frac{J_{0}}{E_{0} v_{\infty}} \approx \frac{\sqrt{2 M r_{\min }}}{v_{\infty}}, \\
& \approx 4 \mathrm{au} \times\left(\frac{r_{\min }}{100 M}\right)^{1 / 2}\left(\frac{M}{10^{3} \mathrm{M}_{\odot}}\right)\left(\frac{10 \mathrm{~km} / \mathrm{s}}{v_{\infty}}\right)
\end{aligned}
$$

For a $M=10^{3} \mathrm{M}_{\odot} \mathrm{BH}$, the impact parameter is in general greater than 1 au for a close fly-by with $r_{\text {min }} \leq 100 M$ when 
$v_{\infty} \leq 10 \mathrm{~km} / \mathrm{s}$. As the scattering cross section is proportional to the square of $b$, lower velocities and a larger BH's mass will lead to larger scattering cross sections.

While young pulsars are high-speed stars (a mean velocity of $\sim 450 \mathrm{~km} / \mathrm{s}$ ) that could probably escape the galactic potential (Lyne \& Lorimer 1994), old pulsars (some of which are MSPs) that have undergone accretion processes are believed to move with much smaller speed, and reach equilibrium during their interactions with the environments. The mean velocity of MSP is generally believed to be around $100 \mathrm{~km} / \mathrm{s}$ (Lyne et al. 1998; Hobbs et al. 2005; Malov \& Baurov 2007; Gonzalez et al. 2011) while some expect lower values (Cordes \& Chernoff 1997; Hooper et al. 2013).

In this work, we consider a typical speed $v_{\infty}=5-10 \mathrm{~km} / \mathrm{s}$ (GCs), $50 \mathrm{~km} / \mathrm{s}$ (small galaxies) and $100 \mathrm{~km} / \mathrm{s}$ (large galaxies) for the MSP. We also consider unrealistic cases with $v_{\infty}=0.1 c$ and $c / 3$ for comparison. The central intermediate mass $\mathrm{BH}$ is set to be of mass $M=10^{3} \mathrm{M}_{\odot}$, but the results are applicable for more massive BHs as well (with an approximate dependency on the mass ratio $m / M$ ). We mainly consider a close encounter of $10 M<r_{\min }<200 M$, but extend the study to a larger parameter space to explore the full extent of observational possibilities.

We notice that, when comparing the results of geodesic motion with spin-coupled motions, there are subtle differences in comparing orbits with the same $E_{0}, J_{0}$ and orbits with the same $p$ (semi-latus rectum), $e$ as pointed out by Bini et al. (2017). Here, we choose to compare orbits with the same $E_{0}, J_{0}$ defined at past infinity, because under the influence of spin, these two values are no longer conserved due to the break down of symmetry. The reason is that, if an MSP is found on a hyperbolic orbit approaching the $\mathrm{BH}$ in the weak field, the motion under the geodesic equation and the motion under the MPD equations are indistinguishable if the MSP has the same $E_{0}$ and $J_{0}$ at past infinity. But orbits with the same $p, e$ under different EOMs are distinguishable even in the weak field, and therefore these orbits will not be considered to be the same. Hence the difference in the predicted orbits under different EOMs lacksufficient physical meaning.

\section{SPIN-ORBIT COUPLING AND SPIN-CURVATURE COUPLING}

\subsection{Relativistic hyperbolic orbit}

When a test object has $E>1$, it follows a hyperbolic orbit around the central massive $\mathrm{BH}$, in which its high angular momentum prevents it from being captured. Fig. 1 shows the geometry of such a system, when the orbit is confined to the equatorial plane. The equatorial hyperbolic orbit is symmetric with respect to the periapsis (i.e. $\phi_{-\infty}=-\phi_{+\infty}$ ), when spin's effeets are ignored. The deflection angle is defined as $\Delta \phi=2 \phi_{\infty}-\pi$, where $\phi_{\infty}$ is shown in Fig. 1 . With the help of conserved values ( $E$ and $L_{z}$ ), the hyperbolic-like orbit of a spinning object scattered by a Schwarzschild BH (Bini \& Geralico 2017) or a Kerr BH (Bini et al. 2017) can be described analytically up to linear order in spin. The azimuthal angle at infinity takes the form:

$\phi_{\infty}=\phi_{0}\left(\chi_{\max }\right)+\hat{s} \phi_{\hat{s}}\left(\chi_{\max }\right)$,

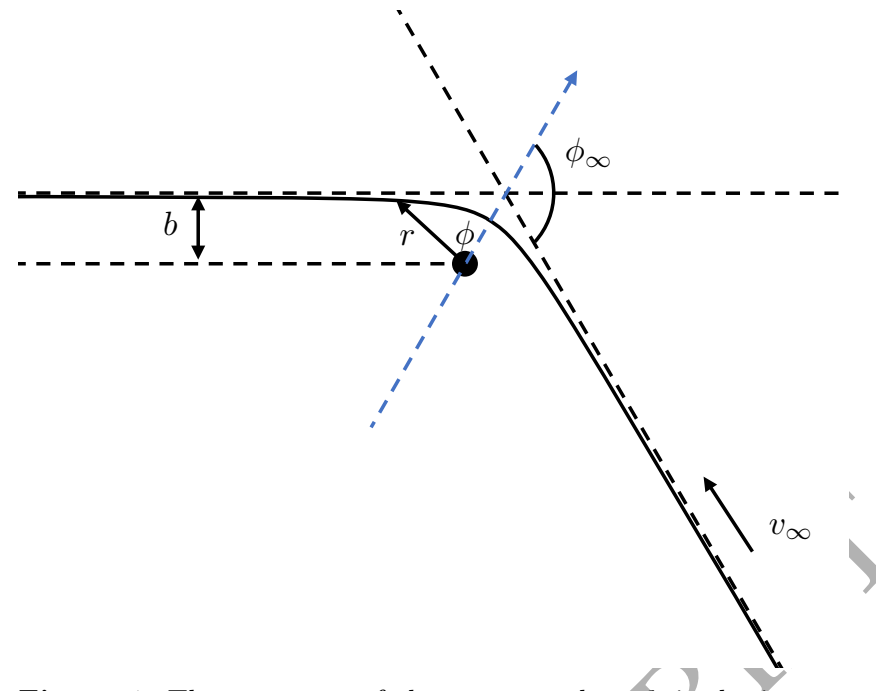

Figure 1. The geometry of the system, where $b$ is the impact parameter and $\phi_{\infty}$ is the angle between the position vector at periapsis and position vector at $t \rightarrow \pm \infty$. When this curve represents geodesic motion, the impact parameter is a constant before and after the scattering. The scattering angle is also symmetric.

where $\phi_{0}(\chi)$ and $\phi_{\hat{s}}(\chi)$ are defined in Eq. (34) of Bini et al. (2017). Note that since the analytical formula that is quadratic in spin is not available, we compare our numerical results (which is quadratic in spin) with Bini's formula at linear order to perform a consistency check.

An example of the deflection angle is shown in Fig.2. Particles with larger $v_{\infty}$ have larger inertia, and therefore are more difficult to be deflected. They follow orbits with much larger eccentricity. Examples of such particles include photons, neutrinos and high energy electrons.

For stars with $v_{\infty} \ll 1$, the unit energy of the test object and the orbit eccentricity are both close to 1 . The eccentricity for such orbits is approximately (exact for Newtonian cases)

$e \approx 1+\frac{r_{\min }}{M} \frac{v_{\infty}^{2}}{c^{2}}$

when $v_{\infty} \leq 100 \mathrm{~km} / \mathrm{s}$ (for any $v_{\infty}$ in Newtonian cases). For such small eccentricity, Newtonian gravity predicts a $\Delta \phi \approx \pi-2 \sqrt{2(e-1)}$, assembling that of an elongated ellipse with infinity semi-major axis. General relativistic effects, in general, increase the deflection angle in the form of periapsis advance. Both the relativistic correction and the spin correction to the deflection angle are greater for smaller eccentricity, making slow speed objects better laboratories for testing the general relativistic effects.

Currently, the formula for the deflection angle of a spinning test object around a Kerr BH is derived for cases where the spin is aligned (or anti-aligned) with the spin of $\mathrm{BH}$ and orbital angular momentum, in which cases, the orbits are confined to the equatorial plane. For non-equatorial orbits, the formula for the deflection angle even for geodesic orbits remains unclear.

When comparing an analytic prediction with numerical simulation results, it is impossible to integrate the orbit to infinity for the MPD equations, and in practice different methods are used to bypass this problem. For example, Damour et al. (2014) compare the results at around 


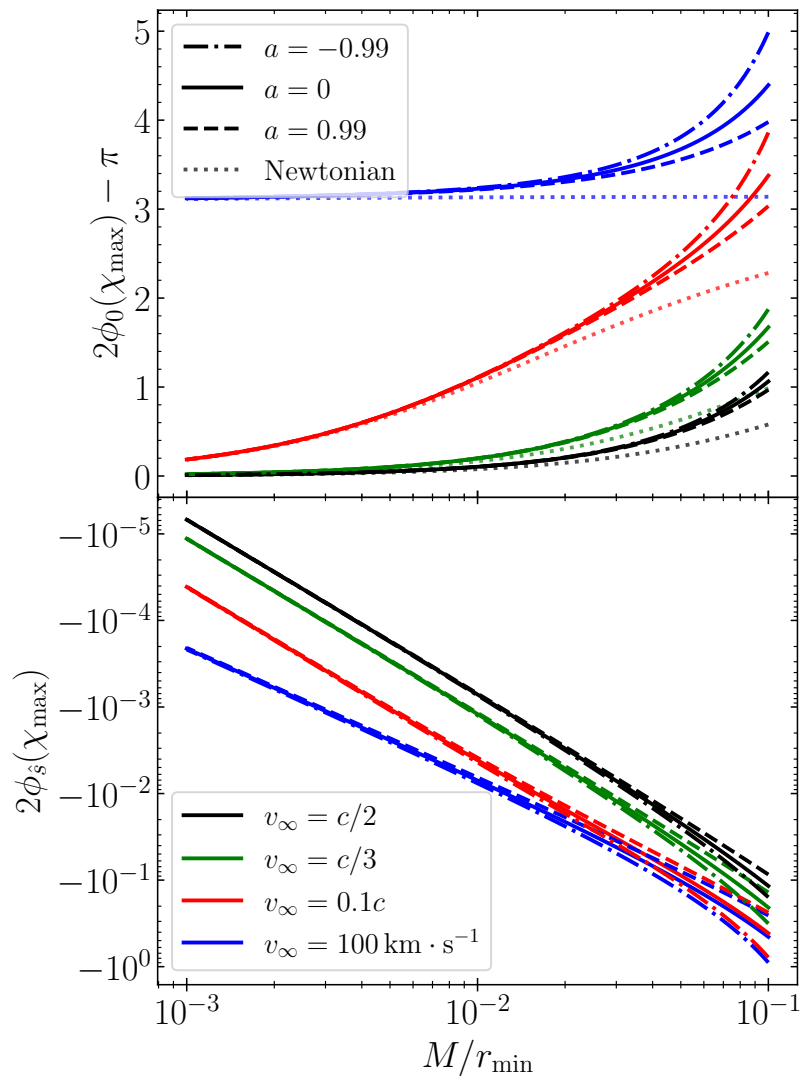

Figure 2. The deflection angle for a time-like test object on geodesic motion (upper panel) and the linear order correction due to spin (lower panel). In the upper (lower) panel, the solid line from top to bottom (bottom to top) corresponds to test objects with $E_{0} \equiv 1 / \sqrt{1-v_{\infty}^{2}}$ where $v_{\infty}=100 \mathrm{~km} / \mathrm{s}, 0.1 c, c / 3$ and $c / 2$, respectively. The angular momentum $J_{0}$ is determined by $v_{\infty}$, $r_{\min } / M$ and $a / M$. The dashed line and dash-dotted line represent $a / M=0.99$ and $a / M=-0.99$, respectively.

$r_{\mathrm{f}} \approx r_{0}=10^{4} M$. In this study, we integrate the MPD equations of motion from $r_{0}=10^{4} M \approx r_{\mathrm{f}}$, and integrate geodesic equations from $r_{-\infty}$ to $r_{0}$ and then $r_{\mathrm{f}}$ to $r_{+\infty}$, assuming the effects of spin become negligible in weak field. Note that we compare orbits for spinning and non-spinning test objects with the same $E_{0}$ and $J_{0}$ at past infinity. Therefore, the spin's correction to the deflection angle is not simply $2 \hat{s} \phi_{\hat{s}}\left(\chi_{\max }\right)$, but rather defined as:

$\Delta \phi_{\text {spin, } \mathrm{A}}=2 \phi_{0}\left(e^{\prime}, p^{\prime}\right)+2 \hat{s} \phi_{\hat{s}}\left(e^{\prime}, p^{\prime}\right)-2 \phi_{0}(e, p)$,

where $e$ and $p$ correspond to the geodesic for given set of $E_{0}$ and $J_{0}$, and $e^{\prime}$ and $p^{\prime}$ differ from $e$ and $p$ at linear order in spin. The relation can be found in Appendix. A.

The geodesic equations can be written in an alternative form for particles confined to the equatorial plane:

$$
\begin{aligned}
& \phi_{ \pm \infty}=\phi_{\mathrm{i}} \pm \int_{r_{\mathrm{i}}}^{\infty} \frac{\Phi_{r}(r)+L_{z}-a E}{\sqrt{R(r)}} \mathrm{d} r, \text { with } \\
& R(r)=\left(E\left(r^{2}+a^{2}\right)-a L_{z}\right)^{2}-\Delta\left(r^{2}+\left(L_{z}-a E\right)^{2}+Q\right), \\
& \Phi_{r}(r)=\frac{a}{\Delta}\left(E\left(r^{2}+a^{2}\right)-a L_{z}\right),
\end{aligned}
$$

where the upper (lower) sign is for particles that are moving to (from) infinity. $\phi_{\mathrm{i}}$ and $r_{\mathrm{i}}$ are "initial condition" of the integrand, which are $\phi_{\mathrm{f}}$ and $r_{\mathrm{f}}$ for $\phi_{+\infty}, \phi_{0}$ and $r_{0}$ for $\phi_{-\infty}$.

When the spin of MSP is not aligned or anti-aligned with the orbital angular momentum, the MSP is not restricted to move in the equatorial plane, the following alternative form of geodesic equations are used to evaluate $\theta_{ \pm \infty}$ :

$$
\begin{aligned}
& \int_{r_{\mathrm{i}}}^{\infty} \frac{\mathrm{d} r}{\sqrt{R(r)}}= \pm \int_{\cos \theta_{\mathrm{i}}}^{\cos \theta_{\infty}} \frac{\mathrm{d}(\cos \theta)}{\sqrt{\Theta(\cos \theta)}}, \text { with } \\
& \Theta(\cos \theta)=Q-\left(Q+a^{2}\left(1-E^{2}\right)+L_{z}^{2}\right) \cos ^{2} \theta+a^{2}\left(1-E^{2}\right) \cos ^{4} \theta
\end{aligned}
$$

where the upper (lower) sign is for $\mathrm{d} \theta / \mathrm{d} r<0(\mathrm{~d} \theta / \mathrm{d} r)>0$ ) , and $\theta_{\mathrm{i}}$ are the initial condition of the integrand, which is $\theta_{\mathrm{f}}$ for evaluating $\theta_{+\infty}$, and $\theta_{0}$ for evaluating $\theta_{-\infty}$. For the azimuthal direction, the values at infinities are

$$
\begin{gathered}
\phi_{ \pm \infty}=\phi_{\mathrm{i}} \pm \int_{r_{\mathrm{i}}}^{\infty} \frac{\Phi_{r}(r)-a E}{\sqrt{R(r)}} \mathrm{d} r \\
-\operatorname{Sign}\left(\frac{\mathrm{d} \theta}{\mathrm{d} \tau}\right) \int_{\cos \theta_{\mathrm{i}}}^{\cos \theta_{\infty}} \frac{\Phi_{\theta}(\cos \theta)}{\sqrt{\Theta(\cos \theta)}} \mathrm{d}(\cos \theta), \text { with } \\
\Phi_{\theta}(\cos \theta)=\frac{L_{z}}{1-\cos ^{2} \theta}
\end{gathered}
$$

where $\mathrm{d} \theta / \mathrm{d} \tau$ is the value at the "initial condition". Due to the effect of spin, the hyperbola is no longer symmetric, and therefore $\phi_{-\infty} \neq-\phi_{+\infty}$, and the deflection angle becomes $\Delta \phi=\phi_{+\infty}-\phi_{-\infty}-\pi$. We define $\Delta \phi_{\text {spin }}=\Delta \phi_{\lambda=1}-\Delta \phi_{\lambda=0}$ to be the correction to the deflection angle due to spin.

\subsection{Validation of the numerical solution}

We solve the MPD equation with a 21-stage 10th order Runge-Kutta scheme with an embedded 9th order method to control the time step ${ }^{2}$. To validate our calculation, the conservation of constants (and semi-constants) are checked for the parameter space of our interest. Fig. 3 shows the maximum violation of the these constants (and semi-constants) during the evolution from $10^{4} M \rightarrow r_{\min } \rightarrow 10^{4} M$ with different EOMs (with or without spin-coupling and quadrupole coupling forces) for systems with $v_{\infty}=10 \mathrm{~km} / \mathrm{s}$ and $J_{0}=$ $J_{0}\left(r_{\min }=10 M\right)$. The parameters of the system cover different spin magnitudes ( $\hat{s}$ from $5.68 \times 10^{-6}$ to $5.68 \times 10^{-2}$ ) and different orientations of the spin axis. The energy, angular momentum, spin magnitude and a further constant $s_{\mu} p^{\mu}$ are all conserved with ratio error $<2 \times 10^{-14}$ for all three different EOMs.

Fig. 4 shows the maximum variation for the semiconstants, including the Rudiger constant $K$, the modified mass $m_{J}$ and the dynamical mass $m$. The dynamical mass $m$ is a constant when $C_{Q}=0$ or $\lambda=0$, and vary as $O\left(s^{2}\right)$ when $C_{Q} \neq 0$. As argued in the previous section, the Rudiger constant is not strictly conserved, but vary as $O\left(s^{2}\right)$ when the orbit does not follow geodesic, and vary as $O\left(s^{3}\right)$ when

2 The coefficients are obtained by Peter Stone and are shown in http://www.peterstone.name. 
the spin is aligned (or anti-aligned) with the orbital angular momentum. The mass-like definition $m_{J}$ varies as $O\left(s^{4}\right)$ when $C_{Q} \neq 0$.

Further, the deflection angles for cases with spin being parallel to the orbital angular momentum are compared for our numerical results with the linear-in-spin analytical formula. The spin will introduce an additional shift of periapsis and change the deflection angle at spatial infinity. In the lower panel of Fig. 4, the difference in two deflection angles, i.e. $\Delta \phi_{\mathrm{N}}$ and $\Delta \phi_{\mathrm{A}}$, are shown for different magnitudes of spin.

When $\lambda=0$ and $C_{Q}=0$, the two formulae deviate by $<10^{-9} \mathrm{rad}$, for systems with $v_{\infty}=10 \mathrm{~km} / \mathrm{s}$ and $J_{0}=J_{0}\left(r_{\min }=\right.$ $10 \mathrm{M})$. The value $10^{-9} \mathrm{rad}$ is the limit of accuracy for double precision, for systems with $v_{\infty}=10 \mathrm{~km} / \mathrm{s}$. A discussion of this accuracy is included in Appendix B. When $\lambda=1$ and $C_{Q} \neq 0$, the difference comes from a combination of numerical error and second-order spin effects. The difference varies as $O\left(s^{2}\right)$ in the lower panel of Fig. 4.

When $180^{\circ}>\theta_{\mathrm{S}}>0^{\circ}$, the MSP is in fact not confined to the equatorial plane, and therefore the analytical formula Eq. 19 are not exact solutions even at linear order. Nevertheless, the lines with triangle and circle markers in the lower panel of Fig. 4 indicate that the analytical formula are still good approximations at linear order.

The variation in deflection angle (as well as periapsis shifts) is mainly due to the force in the radial direction, and therefore is solely contributed by the component of spin that is parallel to orbital angular momentum (see, for example, Eq. 2.2c of Kidder 1995).

\subsection{Motions of MSP at infinity}

Fig. 5 shows the deflection angles for systems with different velocities (i.e. different $E_{0}$ ), different $J_{0}$ and spin directions. The effects of spin favour a system with small $v_{\infty}$ against a system with relativistic speeds. As shown in the figure, the spin's correction to deflection angle of slow-speed test objects can be larger than those of relativistic $v_{\infty}$ by about 10 times. Combining the information of the scattering cross sections, MSPs with small velocities (e.g. $5-10 \mathrm{~km} / \mathrm{s}$ ) are favoured against higher speed MSPs as they have smaller inertia and are easier to be scattered; the scattering cross section is much larger (e.g. $b_{v_{\infty}=10 \mathrm{~km} / \mathrm{s}} / b_{v_{\infty}=100 \mathrm{~km} / \mathrm{s}} \approx 10$ ); and the effects of spin are more prominent. The third point can be visualised from both Fig. 2 and Fig. 5. Smaller $v_{\infty} \ll$ leads to larger $\phi_{\hat{s}}\left(\chi_{\max }\right)$ and hence larger $\Delta \phi_{\text {spin, } \mathrm{A}}$.

As shown in the upper panel of Fig. 5 , when the MSP has $v_{\infty} \ll c$ and when $\theta_{\mathrm{S}}$ is not far from $0^{\circ}$ or $180^{\circ}$, the deflection angle can be well approximated by

$\Delta \phi_{\text {spin }} \approx c_{\phi} \cos \theta_{\mathrm{S}}\left(\frac{1 \mathrm{~ms}}{P_{\mathrm{ns}}}\right)\left(\frac{10^{3} \mathrm{M}_{\odot}}{M}\right)$,

where $c_{\phi}$ is a function of $r_{\min }$ and $a$.

When $180^{\circ}>\theta_{\mathrm{S}}>0^{\circ}$, the orbital plane is tilted around. The motion of the pulsar is usually referred to as the out-of-plane motion. Such motions have been studied extensively in elliptical orbits (see e.g. Singh et al. 2014; Keresztes \& Mikóczi 2019). In hyperbolic scatterings, such out-of-plane motions are also observed. The motion in $\theta$ or $z$ direction mainly depends on the components of spin that is perpendicular to orbital angular momentum. The coupling

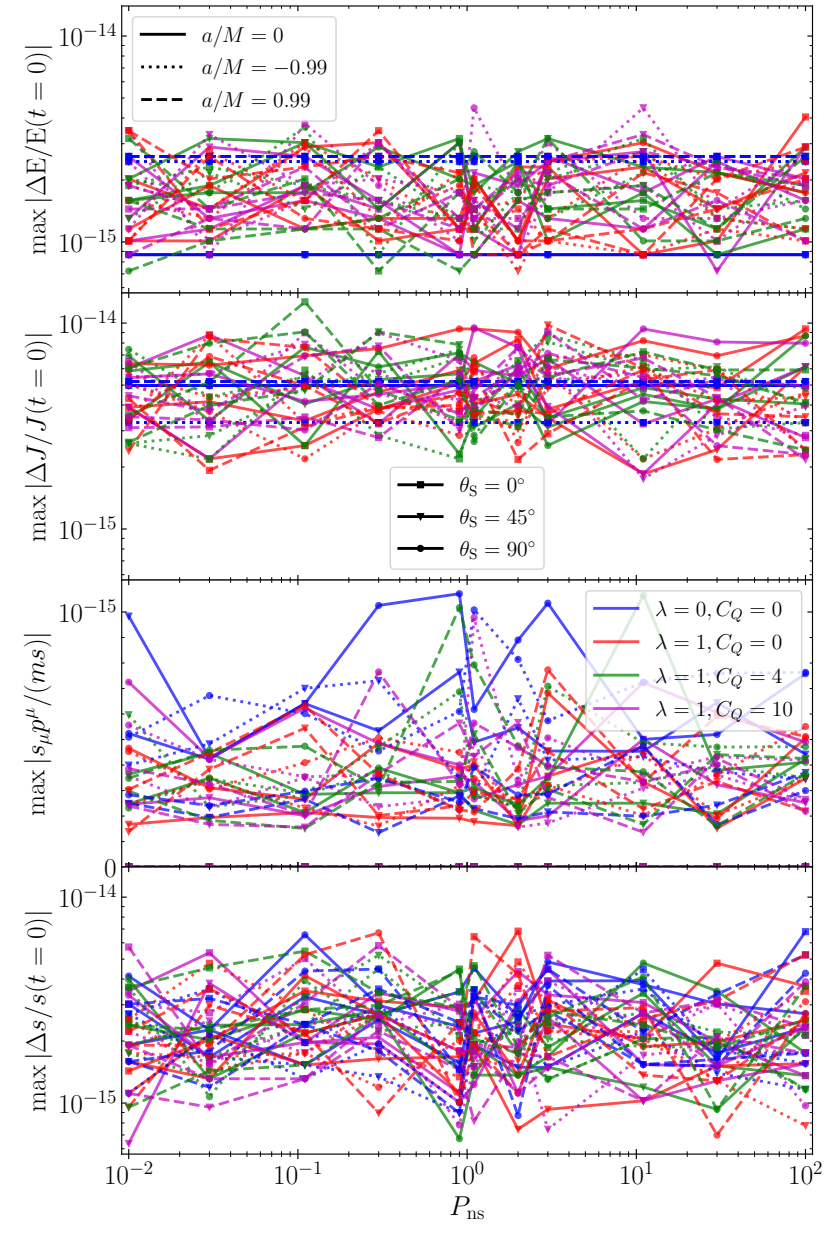

Figure 3. The system considered here is a (unrealistic) pulsar with period $P_{\mathrm{ns}}, m=1.5 \mathrm{M}_{\odot}, v_{\infty}=10 \mathrm{~km} / \mathrm{s}$ and $J_{0}=J_{0}\left(r_{\min }=10 M\right)$ flying by a massive $\mathrm{BH}$ with $10^{3} \mathrm{M}_{\odot}$ and $a / M=0,-0.99,0.99$. The figure shows the maximum difference of the constants compared with their initial values for $E, J, s_{\mu} p^{\mu}$ and $s$. The value $s_{\mu} p^{\mu}$ is zero for TD condition and remains less than $2 \times 10^{-5} \times m \times s$ during the scattering process.

between orbital angular momentum and spin allows for the orbit to wobble, and therefore, when MSP goes to infinity, $\theta_{+\infty}$ deviates from $\theta_{-\infty}=\pi / 2$, and such deviation is shown in Fig. 5 and Fig. 6 , in which we define $\Delta \theta_{\text {spin }} \equiv \theta_{+\infty, \lambda=1}-\pi / 2$, which is the correction of deflection angle in $\theta$ direction due to spin. This deflection angle for orbits with different parameters is shown in the lower panel of Fig. 5 and Fig. 6 . The deflection angle can be approximated by:

$\Delta \theta_{\text {spin }} \approx c_{\theta} \sin \theta_{\mathrm{S}} \sin \left(\phi_{\mathrm{S}}-\phi_{\mathrm{ref}}\right)\left(\frac{1 \mathrm{~ms}}{P_{\mathrm{ns}}}\right)\left(\frac{10^{3} \mathrm{M}_{\odot}}{M}\right)$,

where $\phi_{\text {ref }}$ corresponds to the value of $\phi_{\mathrm{S}}$ when $\Delta \theta_{\text {spin }}=0$ in Fig. 6. The coefficient $c_{\theta}$ is also a function of $r_{\min }$ and $a$. The values of $c_{\theta}, c_{\phi}$ and $\phi_{\text {ref }}$ are summarised in Fig. 7. The value of $\phi_{\text {ref }}$ is related to the the position of periapsis $\phi_{\text {per }}$, which describes the orientation of the hyperbola, and it gets closer to $\phi_{\text {per }}-\pi$ for larger $r_{\min } / M$. The slope of $\log \left(c_{\theta}\right)$ versus $\log \left(r_{\min } / M\right)$ and $\log \left(c_{\phi}\right)$ versus $\log \left(r_{\min } / M\right)$ are all smaller than -1.4 , implying that this effect is highly relativistic, and is important only for very close fly-bys.

When the central BH is spinning, the spin of the $\mathrm{BH}$ will 


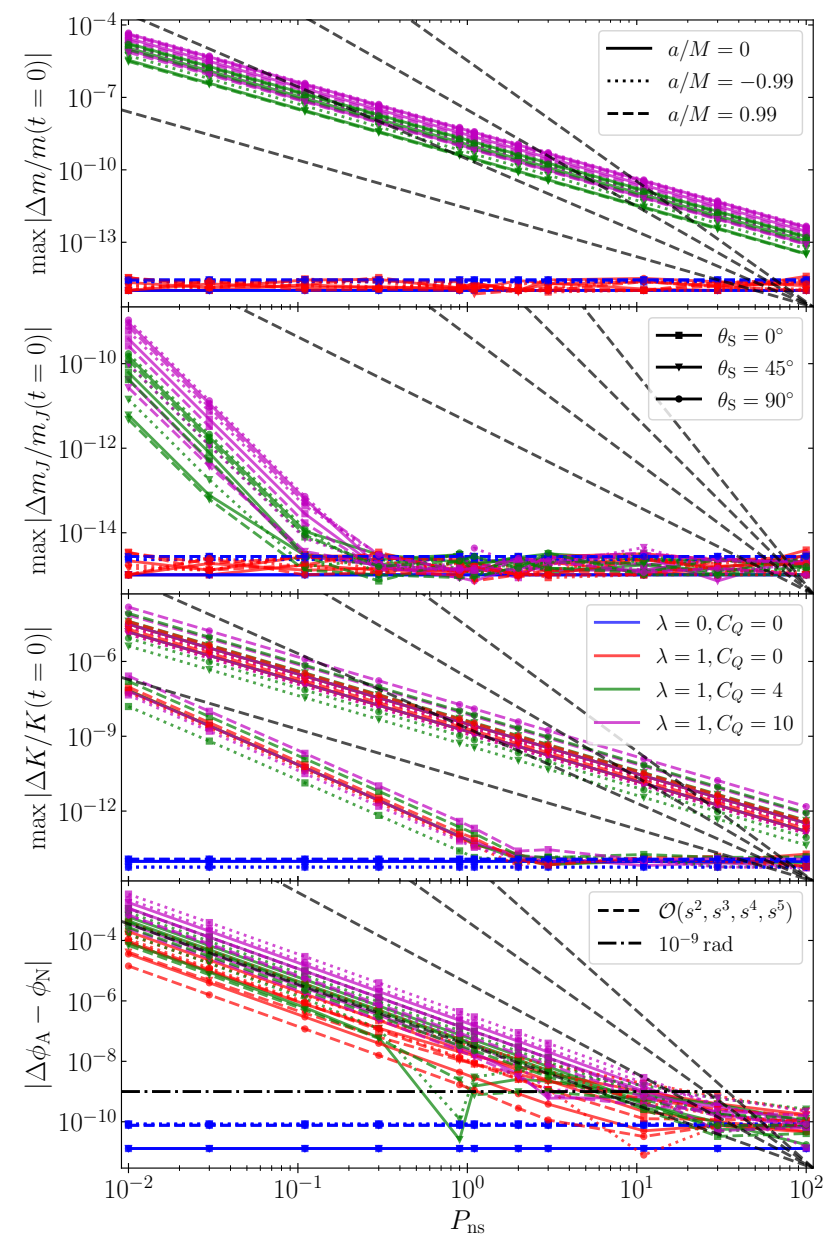

Figure 4. The figure shows the maximum variation of semiconstants: dynamical mass $m \equiv \sqrt{-p^{\mu} p_{\mu}}$, the modified mass $m_{J}$, the Rudiger constant $K$ and the difference between numerical deflection angle $\Delta \phi_{\mathrm{N}}$ (calculated by Eq. 22 and Eq. 24) and $\Delta \phi_{\mathrm{A}} \equiv 2 \phi_{0}\left(\chi_{\max }\right)+2 \hat{s} \phi_{\hat{s}}\left(\chi_{\max }\right)-\pi$. The black dashed lines in each panel represent the reference lines which are $\propto s^{2}, s^{3}, s^{4}, s^{5}$.

introduce an additional shift to the periapsis and $\phi_{ \pm \infty}$. Even a moderate spin of the $\mathrm{BH}$ (e.g. $a \approx 0.5 \mathrm{M}$ ) would greatly exceed the spin of the MSP and therefore the spin-orbit coupling will be dominated by the spin of the BH. When the BH's spin is in the same direction with the angular momentum, the spin-orbit coupling would introduce an additional force in the opposite direction of Newtonian gravity (see, e.g. Eq. 2.2c of Kidder 1995), preventing the test object from getting too close to the $\mathrm{BH}$, and therefore reduces the deflection angle. As readily shown in Fig. 6, and Fig. 2, the retrograde motion has larger deflection angle compared with a comparable prograde motion.

Because the spin of the $\mathrm{BH}$ dominates, the effects of the MSP's spin can be seen as a perturbation to the geodesic around the Kerr BH. Therefore, the effects of the MSP's spin are still approximately symmetrical for $\theta_{\mathrm{S}}$ and $180^{\circ}-\theta_{\mathrm{S}}$. In fact, both $\Delta \theta_{\text {spin }}$ and $\Delta \phi_{\text {spin }}$ follow the same relation as Eq. 25 and Eq. 26. The deflection angles in both directions are the largest for retrograde motion, and smallest for prograde motion.

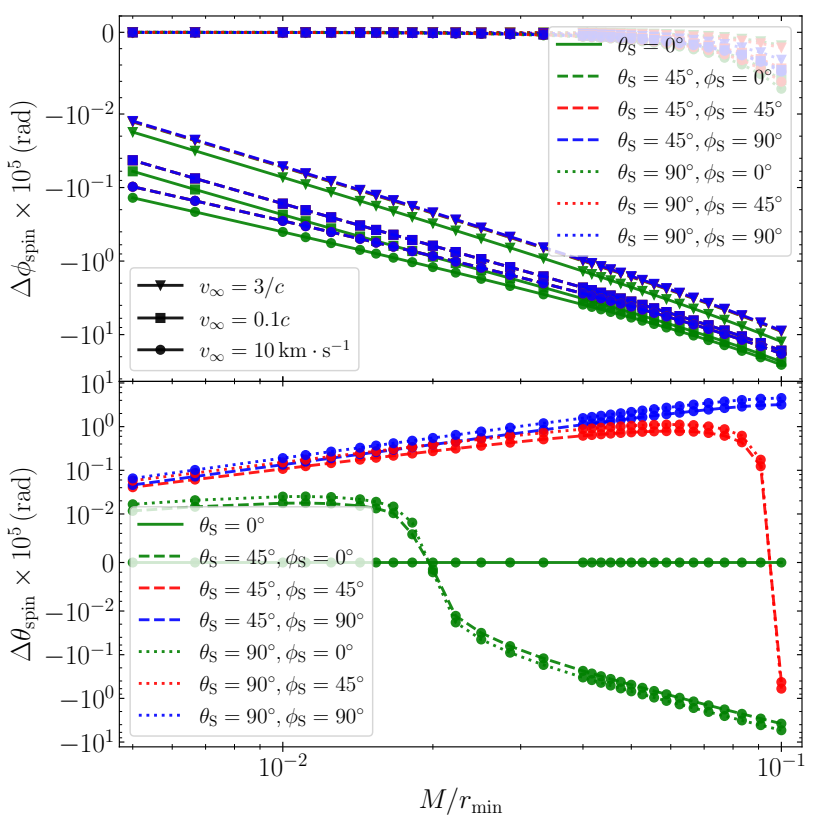

Figure 5. The corrections to the two deffection angles (i.e. $\Delta \phi_{\text {spin }}$ and $\left.\Delta \theta_{\text {spin }}\right)$ due to the spin the of MSP with different spin orientations. The spin axes of the MSPs are either perpendicular to the equatorial plane $\left(\theta_{\mathrm{S}}=0^{\circ}\right.$, solid lines), or tilted at $\theta_{\mathrm{S}}=45^{\circ}$ (dashed lines) or $\theta_{\mathrm{S}}=90^{\circ}$ (dotted lines) with respect to the $\hat{z}$. The $\mathrm{BH}$ is non-spinning and has mass $M=10^{3} \mathrm{M}_{\odot}$. The upper panel shows the MSP with different energies (i.e. $v_{\infty}$ ) and angular momenta. The lower panel shows the MSP with $v_{\infty}=10 \mathrm{~km} / \mathrm{s}$ and different angular momenta.

\subsection{Detectability of the deviation from geodesic} For an $M=10^{3} \mathrm{M}_{\odot} \mathrm{BH}$, a typical deviation in deflection angle is about $\sim 2 \times 10^{-5}$ rad for a close fly-by $\left(r_{\min } \approx 20 M\right)$. This deviation in angles corresponds to a spatial deviation of $\sim 30 \mathrm{~km}$ when $r=1000 \mathrm{M}$, which could introduce an $0.1 \mathrm{~ms}$ difference in the pulse-arrival-time, different from that of a pulsar following geodesic. This is within the precision limit of current pulsar timing technique (see footnote 8 of Li et al. 2019). In general, assuming the detectable limit to be $0.1 \mathrm{~ms}$ (i.e. $30 \mathrm{~km}$ ), if a spinning MSP is found to be flying towards the $\mathrm{BH}$ at $r=10^{4} \mathrm{M}$, the orbit of this MSP will deviate from the geodesic orbit by $\Delta r=30 \mathrm{~km}$ after a certain observational time. This observational time for pulsars with different orbital parameters are shown in Fig. 8. The corresponding radial distance between the MSP and the $\mathrm{BH}$ is shown in Fig. 11.

If the pulsar is an MSP that will undergo a close fly-by $\left(r_{\min }<100 M\right)$, the observational time is in general smaller than 2 hours for most orientation of the spin axis. If we limit the observational time to be 1 hour, for each possible orientation of the spin axis, there is a maximum $r_{\min } / M$ below which the orbit of the MSP will deviate from the geodesic by $\Delta r \geq 30 \mathrm{~km}$ within 1 hour. The maximum value of $r_{\min } / M$ is shown in the upper panel of Fig. 9. It is clear that $\theta_{\mathrm{S}}=0^{\circ}, 180^{\circ}$ are favoured orientations (with larger $\left.\max \left(r_{\min }\right) / M\right)$, and even when $\theta_{\mathrm{S}}=90^{\circ}$, there are still some favoured directions around $\phi_{\mathrm{S}}=-110^{\circ}$ and $70^{\circ}$. If an MSP is found at $r=10^{4} \mathrm{M}$ moving towards the $\mathrm{BH}$ with random orientation, then $\Omega / 4 \pi=25.8 \%$ of the MSP will have a $0.1 \mathrm{~ms}$ shift in pulse-arrival-time if the pulsar is found to follow an 


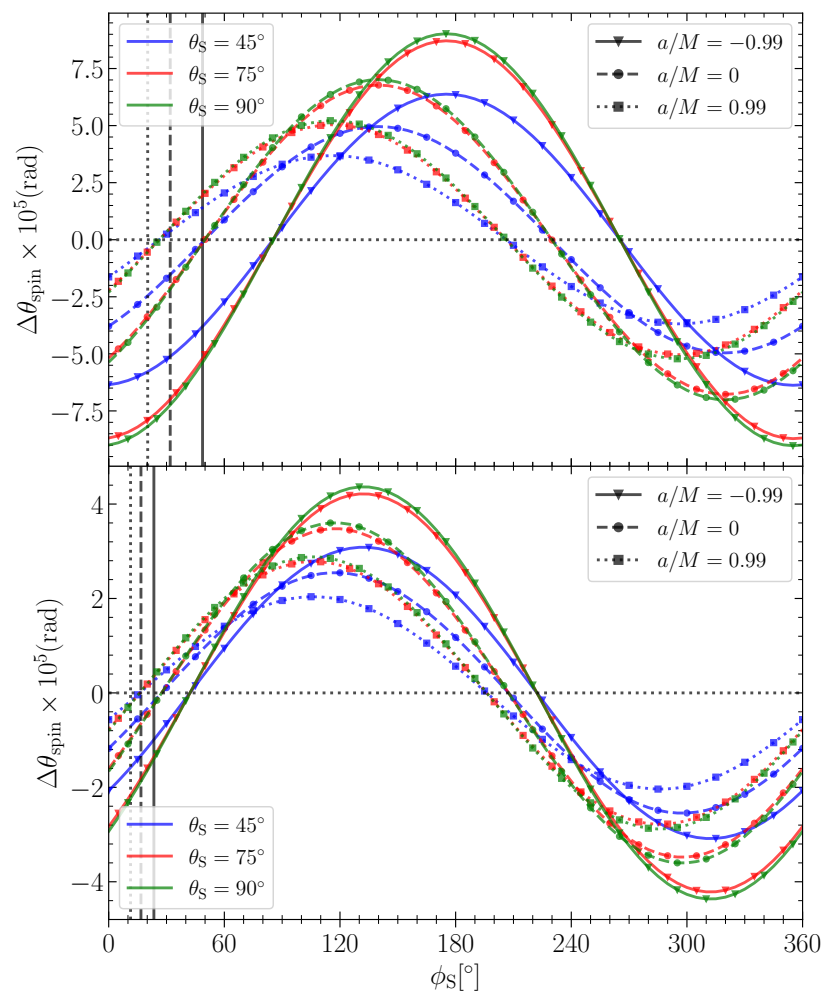

Figure 6. The corrections to the deflection angle of the MSP in $\theta$ direction due to the spin of the MSP. The MSP has $v_{\infty}=10 \mathrm{~km} / \mathrm{s}$ and $r_{\min } / M=10$ (upper panel) or $r_{\min } / M=15$ (lower panel). The $\mathrm{BH}$ is non-spinning and has mass $M=10^{3} \mathrm{M}_{\odot}$. The vertical lines represent the orientation of the orbit (i.e. $\phi_{\text {per }}-\pi$ ).

orbit of $r_{\min } \leq 100 \mathrm{M}$. Note that $r_{\min } \approx 100 \mathrm{M}$ corresponds to a scattering cross-section of $\pi \times(4.2 \mathrm{au})^{2}$ for $M=10^{3} \mathrm{M}_{\odot}$ and $v_{\infty}=10 \mathrm{~km} / \mathrm{s}$.

If we suppose MSPs are scattered into the $\mathrm{BH}$ evenly over some cross section $b_{\mathrm{cs}} \leq r_{\text {inf }}$, then the effective cross section $A$, in which the spin-orbit coupling effects can be observed within one hour after the pulsar reaches $r=10^{4} \mathrm{M}$ during inward motion, is

$A_{a=0}=\int_{0}^{b_{\mathrm{cs}}} \frac{\Omega(b)}{4 \pi} 2 \pi b \mathrm{~d} b \approx \pi \times(3.7 \mathrm{au})^{2}$.

By setting $b_{\mathrm{cs}} \rightarrow \infty$, we can define the effective scattering cross section as

$\pi b_{\mathrm{eff}}^{2} \equiv \int_{0}^{\infty} \frac{\Omega(b)}{4 \pi} \times 2 \pi b \mathrm{~d} b$.

The effective scattering cross sections for $P_{\mathrm{ns}}=1 \mathrm{~ms}, 10 \mathrm{~ms}$, and different observation times are shown in Fig. 10.

Fig. 12 shows the critical $r_{\text {min }}$ for the detection of the MSP spin effects if such an MSP fly by a spinning BH, compared with that of a Schwarzschild BH, for MSP with a few typical $\theta_{\mathrm{S}}$ values between $0^{\circ}-90^{\circ}$. In general, prograde motion requires slightly smaller critical $r_{\text {min }}$ while retrograde motion requires slightly larger $r_{\min }$, and the locations of the peaks approximately correspond to the values $\phi_{\text {ref }} \pm \pi / 2$ at corresponding $r_{\min } / M$.

Another measure of the effects of spin-orbit coupling is the radial distance of the pulsar from the $\mathrm{BH}$ when the deviation from the geodesic reaches $30 \mathrm{~km}$. As shown in Fig. 11, the pulsar will deviate from the geodesic by $30 \mathrm{~km}$ before $r$

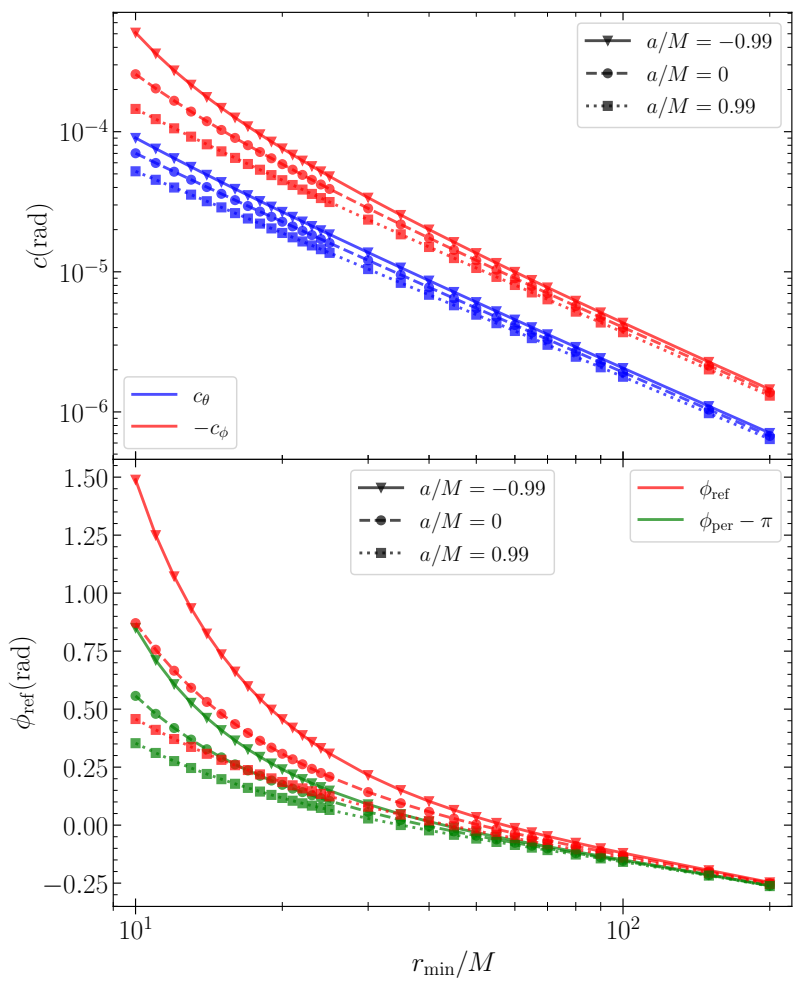

Figure 7. The upper panel shows the value of coefficients $c_{\theta}$ (Eq. 26) and $c_{\phi}$ (Eq. 25) for $10 M<r_{\min }<100 M$ and $a=0, \pm 0.99 M$. The lower panel shows reference angles $\phi_{\text {ref }}$ and the location of periapsis $\phi_{\text {per }}$.

reaches

$$
=1.4 \mathrm{au} \times\left(\frac{M}{10^{3} \mathrm{M}_{\odot}}\right),
$$

much smaller than the influence radius for a $\mathrm{BH}$ between $10^{3}-4 \times 10^{6} \mathrm{M}_{\odot}$. This criterion allows us to ignore the effects of surrounding stars of the MSP's orbit and, therefore, simplify the search for such radio pulses.

Because the deflection angle is inversely proportional to the mass of the $\mathrm{BH}$, the radial distance at which deviation from the geodesic reaches $30 \mathrm{~km}$ is approximately linearly proportionally to the mass of $\mathrm{BH}$, i.e. $r / M$ is approximately constant. The time is, therefore, also linearly proportionally to the mass of $\mathrm{BH}$. Hence an observation of one hour for $M=10^{3} \mathrm{M}_{\odot}$ corresponds to about 4 days for $M=10^{5} \mathrm{M}_{\odot}$ and about 6 months for $M=4 \times 10^{6} \mathrm{M}_{\odot}$. Long time radio observation is possible for important pulsars (see e.g. Weisberg \& Taylor 2005; Ransom et al. 2020), and we do not request the observation data to be continuous in time. For the same $r_{\min } / M$, the impact parameter is linearly proportionally to BH's mass. Therefore, for more massive $\mathrm{BHs}$, the scattering cross section is much larger $\left(\propto M^{2}\right)$. If we assume a toy model that the MSPs are scattered into the BHs evenly within the influence radius (which scales as $\propto M^{0.5-0.6}$ ) with a fixed rate, the event rate for more massive BHs is much larger than those of smaller masses, even though the observation of spin effects in scattering by more massive $\mathrm{BH}$ requires a much longer observational time. A realistic event rate estimate requires detailed modelling of the stellar populations and evolution history of the GC's core 

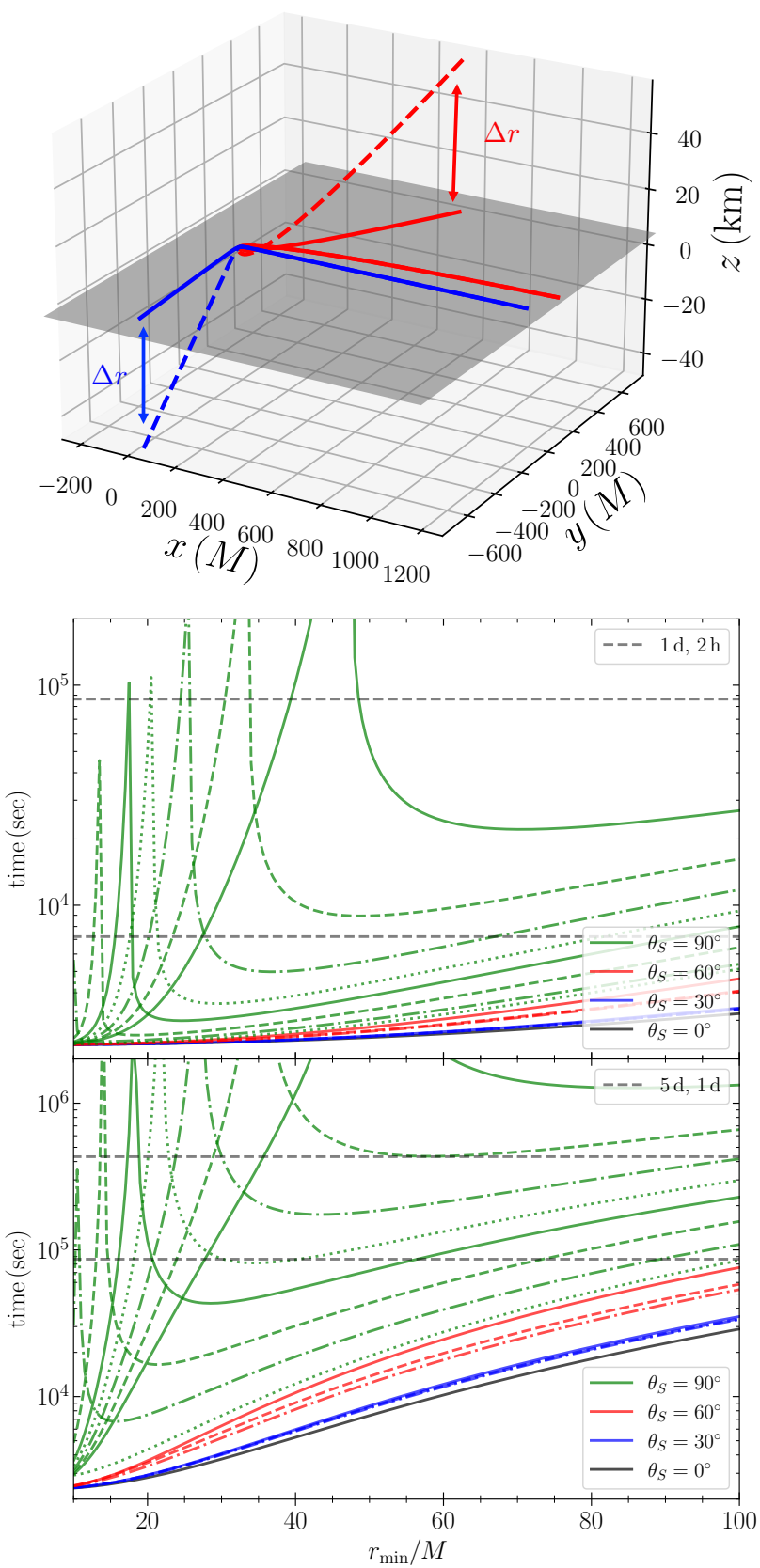

Figure 8. This figure shows the observation time it takes for an MSP to deviate from its geodesic orbit by $30 \mathrm{~km}$, if the MSP is found at $r=10^{4} \mathrm{M}$ approaching the BH. The upper panel is an illustration of the deviation. The red and blue lines are geodesic orbits of different energies and angular momenta, and the dashed lines are the corresponding non-geodesic orbits. The middle panel shows an MSP pulsar $\left(P_{\mathrm{ns}}=1 \mathrm{~ms}\right)$. The lower panel shows a pulsar with period of $10 \mathrm{~ms}$. In both the middle and the lower panels, from top to bottom, the first 8 green lines represent MSPs with $\theta_{\mathrm{S}}=90^{\circ}$ and $\phi_{\mathrm{S}}=0^{\circ}$ (solid), $5^{\circ}$ (dashed), $10^{\circ}$ (dashed-dotted), $15^{\circ}$ (dotted), $20^{\circ}$ (solid), $30^{\circ}$ (dashed), $45^{\circ}$ (dashed-dotted), and $90^{\circ}$ (dotted). The successive 3 red lines represent MSPs with $\theta_{\mathrm{S}}=60^{\circ}$ and $\phi_{\mathrm{S}}=0^{\circ}$ (solid), $45^{\circ}$ (dashed) and $90^{\circ}$ (dashed-dotted), in which the dashed line and dashed-dotted lines almost overlap. The successive 3 blue lines represent MSPs with $\theta_{\mathrm{S}}=30^{\circ}$ and $\phi_{\mathrm{S}}=0^{\circ}$ (solid), $45^{\circ}$ (dashed) and $90^{\circ}$ (dashed-dotted), in which all three lines overlap. The black line at the bottom refers to an MSP with $\theta_{\mathrm{S}}=0^{\circ}$. The horizontal dashed lines represent reference lines of 2 hours and one day in the middle panel, and one day and 5 days in the lower panel. For MSPs, most of the observational times required are below 2 hours for a close fly-by $r_{\min }<100 M$. For a pulsar with period $10 \mathrm{~ms}$, the observational times required are mostly below 2 days.
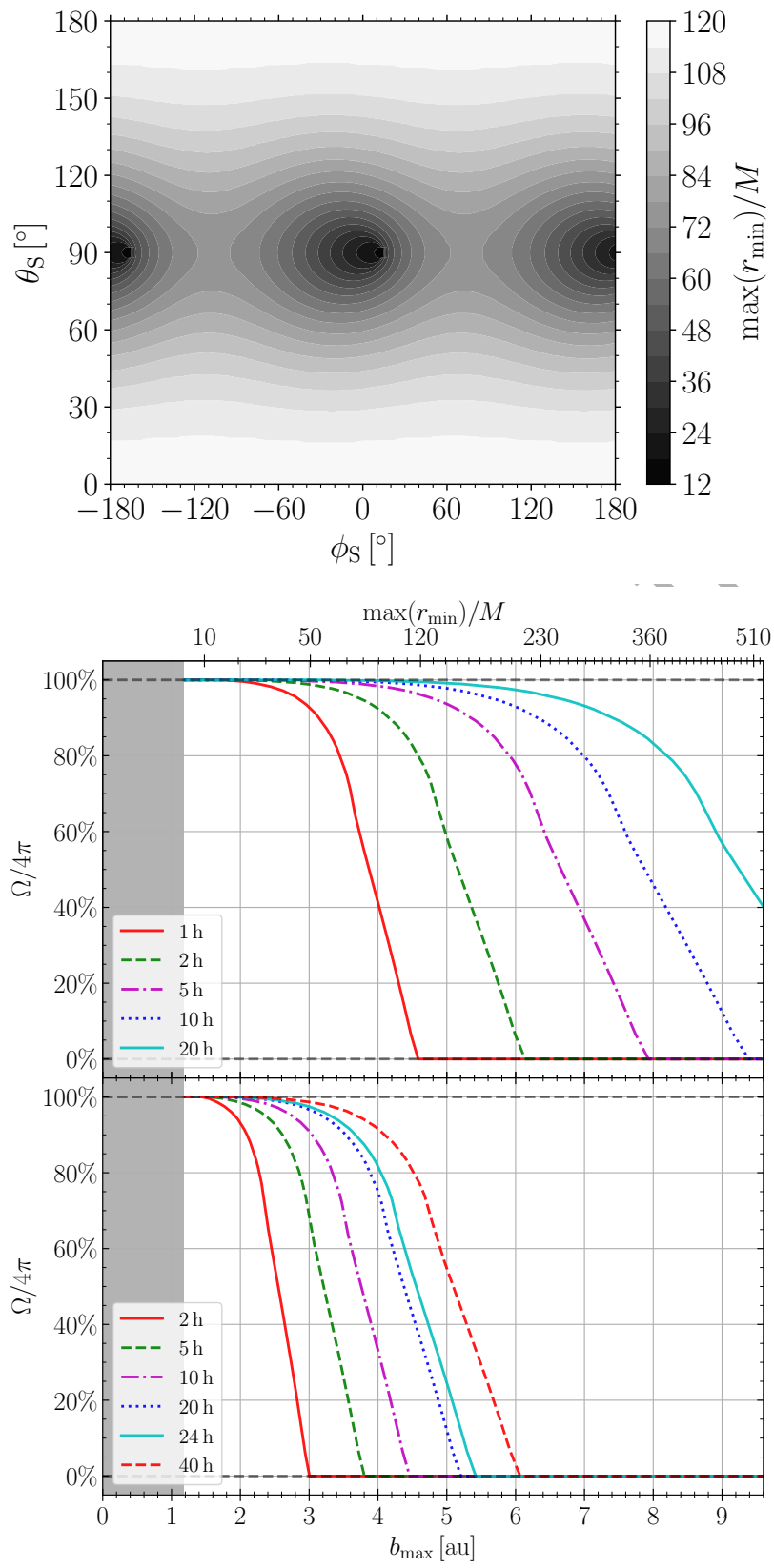

Figure 9. Suppose that an MSP (with $v_{\infty}=10 \mathrm{~km} / \mathrm{s}^{-1}$ ) is found at $r=10^{4} M$ moving towards the $\mathrm{BH}$ with certain orientation (described by $\theta_{\mathrm{S}}$ and $\phi_{\mathrm{S}}$ ), if the deviation from geodesic is required to reach $\Delta r=30 \mathrm{~km}$ within 1 hour of observation time, there is a maximum required $r_{\min } / \boldsymbol{M}$ for this orbit. The upper panel shows the maximum required $r_{\min } / M$ for different orientations of the spin axis. The lower two panels show the possibility that a deviation of $\Delta r=30 \mathrm{~km}$ can be observed within the given observational time, if the orbit has a fixed $r_{\min } / \boldsymbol{M}$, for an MSP (middle panel) and a $10 \mathrm{~ms}$ pulsar (lower panel). The possibility is determined by the orientation of the spin axis of the pulsar, and is therefore given in solid angle $\Omega$. If $\Omega / 4 \pi=1$, the deviation from geodesic can be observed regardless of the orientation of the pulsar, while a value smaller than 1 means that the deviation will be observed only if the pulsar has its spin axis pointing at some preferred directions (in general, parallel and anti-parallel to the orbital angular momentum). The shaded regions in lower panels represent the capture domain if gravitational radiation is ignored. 


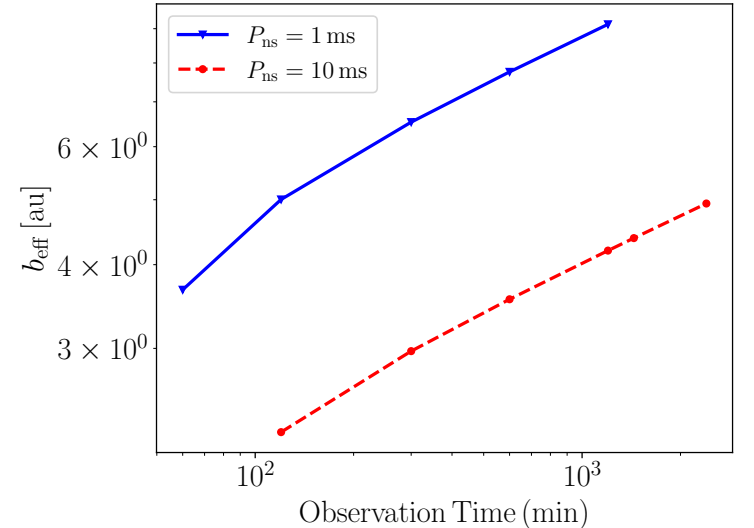

Figure 10. The effective scattering cross sections $b_{\text {eff }}$ for $P_{\mathrm{ns}}=$ $1 \mathrm{~ms}, 10 \mathrm{~ms}$, and different observation times between $1 \mathrm{~h}$ and $40 \mathrm{~h}$. The $b_{\text {eff }}$ is defined in Eq. 28.

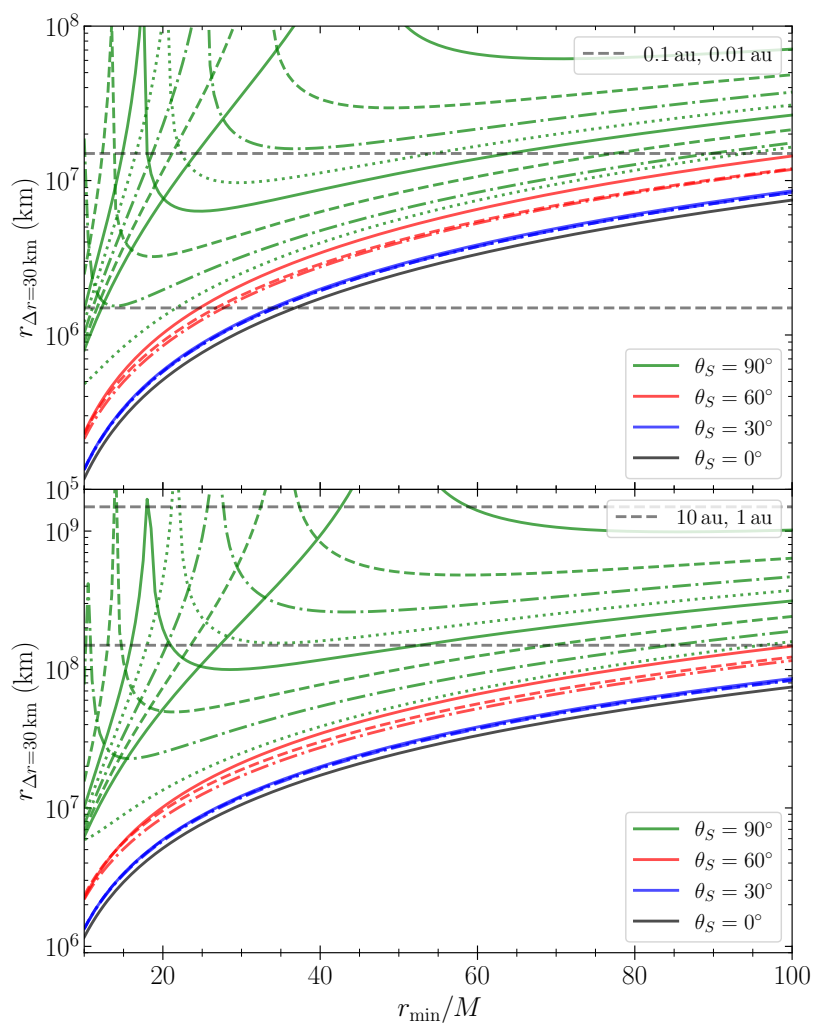

Figure 11. The radial distance of the MSP with the massive BH when $\Delta r=30 \mathrm{~km}$. The upper (lower) panel is for a pulsar with spin period $1 \mathrm{~ms}(10 \mathrm{~ms})$. The parameters of all lines are the same as those in Fig. 8. The $r$ values are linearly proportional to BH's mass. For both the MSP and the $10 \mathrm{~ms}$ pulsar, if they undergo a close fly-by $\left(r_{\min }<100 M\right)$ around the $\mathrm{BH}$, the deviations from the geodesic orbit exceed $30 \mathrm{~km}$ before reaching 1.4 au for most of the spin orientations.

or galactic nuclei, and is beyond the scope of this work. We look forward to future studies on this issue.

If we allow the detector to track one pulsar for a sufficiently long time (say, e.g. one year), then the detectablity of the spin-orbit coupling is greatly enhanced. Even MSPs that undergo a distant fly-by will deviate from the geodesic orbit by $30 \mathrm{~km}$ before the MSP reaches radial infinity. Yet it is computationally expensive to calculate these kinds of

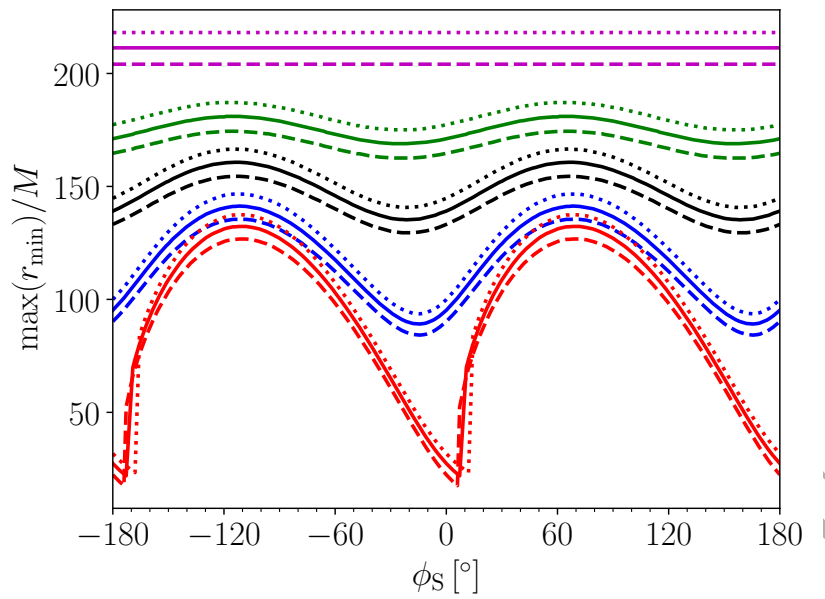

Figure 12. For each assembly of lines, the solid (dashed, dotted) line represent $a=0 M(a=0.99 M, a=-0.99 M)$. Each bundle of lines from the top to the bottom represents $\theta_{\mathrm{S}}$ being $0^{\circ}$ (magenta), $45^{\circ}$ (green), $60^{\circ}$ (black), $75^{\circ}$ (blue), $90^{\circ}$ (red), respectively.

orbits by solving the MPD equations numerically, due to the increasing numerical error as we integrate the orbits to large radial distance. In other words, a search for even a distant fly-by may require taking spin-orbit coupling into account for long term observations.

\section{IMPLICATIONS IN ASTROPHYSICS AND PHYSICS}

4.1 Presence of pulsars around a massive black hole

Each massive galactic spheroid, such as an elliptical galaxy or a bulge of a large spiral galaxy, would host at least one massive nuclear $\mathrm{BH}$. The mass of the nuclear $\mathrm{BH}$ and the stellar dynamics of the host galaxy are correlated, following an empirical M- $\sigma$ relation (Magorrian et al. 1998; Ferrarese \& Merritt 2000; Gebhardt et al. 2000), which implies that massive nuclear BHs reside in massive stellar spheroids. Extrapolating the M- $\sigma$ relation down to the lower mass spheroids, we expect that nuclear BHs of mass $\sim 10^{3}-10^{6} \mathrm{M}_{\odot}$ would be present in the cores of GCs and the bulges of spirals and dwarf galaxies (see Miller \& Hamilton 2002; Reines \& Volonteri 2015; Mezcua 2017). Nuclear BHs were found in the bulges of many late spirals (see e.g. Jiang et al. 2011; She et al. 2017). There are also evidences of nuclear BHs in a number of dwarf galaxies (Reines et al. 2013; Moran et al. 2014; Nguyen et al. 2017), including the compact dwarfs (Voggel et al. 2018). The search of nuclear BHs in GCs have shown promising results (Gerssen et al. 2002; Noyola et al. 2008; Ibata et al. 2009; Feldmeier et al. 2013; Lützgendorf et al. 2015), with an intermediate-massblack hole identified in NGC 6624 (Perera et al. 2017) and 47 Tucanae (Kiziltan et al. 2017).

The dense environment of the GCs facilitates the formation of MSPs (see e.g. Bhattacharya \& van den Heuvel 1991; Manchester 2017). The high stellar encounter rates in the core can facilitate the formation of X-ray binaries, where neutron stars can be spun up by the masstransfer/accretion process (Srinivasan \& van den Heuvel 
1982; Bhattacharya \& van den Heuvel 1991). Indeed, increasing number of MSPs are being found (Lyne et al. 1987; Manchester et al. 1991; Abdo et al. 2013; Freire et al. 2017; Dai et al. 2020), and up to now, 232 MSPs are found among $36 \mathrm{GCs}^{3}$. Population estimation suggests that the actual population of MSPs should be much larger. Constraints from gamma ray observations suggest that 2600-4700 MSPs could reside in Galactic GCs, while radio flux observations suggest that 500-2000 MSPs in Galactic GCs (Fruchter \& Goss 2000), or 12-815 MSPs per GC (see Bagchi et al. 2011). Xray observations suggest that 700 MSPs could reside in Galactic GCs (see Heinke et al. 2005). Large uncertainty exists even for the prediction of an MSP population in a single GC, due to the complicated dynamical evolution history of GCs. Some studies try to find a relation between the MSP population with the stellar encounter rate (Bagchi et al. 2011; de Menezes et al. 2019) or the metalicity (Ivanova et al. 2008; Hui et al. 2010; Bahramian et al. 2013; de Menezes et al. 2019) but the populations are preliminarily estimated from gamma-ray or radio observation. However, the gamma ray luminosity is not necessarily a good probe of the MSP population, as not all MSPs emit gammarays (some might be faint, see e.g. Romani et al. 2011; Smith et al. 2019), and the gamma ray emission in some GCs are dominated by one MSP (Freire et al. 2011). Further, for the current radio luminosity model, the radio frequency is usually above $400 \mathrm{MHz}$ (Bagchi et al. 2011; Calore et al. 2016). A low frequency (115-155 MHz) search indicated that a potential population of MSPs with steep spectrum might have been missed in previous searches (Pleunis et al. 2017).

In our Galaxy, many pulsars are believed to reside in dense nuclei, and MSPs are believed to dominate (Macquart \& Kanekar 2015), although no radio observation has confirmed this hypothesis, except for a magnetar found at $0.1 \mathrm{pc}$ (Rea et al. 2013). The gamma ray excess might be the consequence of this MSP population (Brandt \& Kocsis 2015; Bartels et al. 2016; Fragione et al. 2018; Eckner et al. 2018), although some suggest that MSPs could only account for a small portion (Hooper \& Linden 2016). The MSP population predicted from different models is highly uncertain, ranging from 200 (Chennamangalam \& Lorimer 2014, using the MC method, and over several thousands for Bayesian method), $10^{3}$ (Wharton et al. 2012) to $10^{4}$ (Rajwade et al. 2017) within the inner 1 pc. Nevertheless, the time scale of a close fly-by around Sgr $\mathrm{A}^{*}$ is much longer $(\propto M$, and therefore 4000 times of that of $\left.10^{3} \mathrm{M}_{\odot} \mathrm{BH}\right)$. The scattering cross section will be larger by a factor $10^{6}$ (because $b \propto M^{2}$ for same $r_{\min } / M$, but the dispersion velocity is $\approx 100 \mathrm{~km} / \mathrm{s}$ ). How this affects the event rate and detectablity of a close MSPBH fly-by requires detailed studies in the future.

Because of dynamical friction, these MSPs or their progenitors would sink to the bottom of the gravitational well of the stellar spheroids (de Menezes et al. 2019). The sinking of the neutron stars to the centre of the stellar spheroid also enhances the chance of encounters of them and the nuclear $\mathrm{BH}$, if present, leading to either capture (bounded orbit) or scattering (unbounded orbit).

Finding MSPs in the core of stellar spheroids has nu-

3 A list of pulsars in GCs can be found in

http://www.naic.edu/ pfreire/GCpsr.html. merous prospects and, therefore, has attracted continuous efforts over decades. The MSPs in the Galactic centre could be formed during the local star forming phase (the socalled in situ formation scenario, see e.g. Aharon \& Perets 2015), or could be the heritage of tidally disrupted GCs (Calore et al. 2016; Arca-Sedda et al. 2018; Abbate et al. 2018). The observations of MSP populations in the Galactic centre would provide information regarding the formation history of our Galactic nuclei, and constrain the existing astrophysical models of nuclei stellar cluster. The observation is challenging, given the complex structures in the interstellar medium at the galactic nuclear region, and is beyond the sensitivity of current surveys (Rajwade et al. 2017). The strong scattering and the temporal smearing at low frequencies make it difficult to detect the periodic radio signal (Cordes \& Chernoff 1997; Macquart et al. 2010; Hyman et al. 2019), in particular, for MSPs which are not as luminous as a normal pulsar. Not only is the dispersion measure (DM), about $1000 \mathrm{pc} \mathrm{cm}^{-3}$ (see e.g. Cordes \& Lazio 2002; Yao et al. 2017) much greater than all GCs in the Galaxy. For example, M53, at distance of $\sim 18 \mathrm{kpc}$. much farther away than the Galactic centre, has a DM $\sim 255 \mathrm{pc} \mathrm{cm}^{-3}$ (Kulkarni et al. 1991). Some studies suggest that the DM in the Galactic centre may also be frequency-dependent (Pennucci et al. 2015; Cordes et al. 2016). Understanding the properties of the interstellar medium in the Galactic centre is crucial for the identifying origins of radio sources and for example, analysing the image of Sgr A* using Event Horizon Telescope. Finding a pulsar in the centre of a GC or Galactic nuclei will provide direct measurement of the local gravitational potential. For GCs, it allows us to differentiate between different models (e.g. King Model or Plummer model). The degeneracy between density distribution and surface luminosity can potentially be resolved by this information direct from the inner core. A pulsar in the inner core could also reveal the presence of a BH. For example, using 3 MSPs in NGC 6752, a large central mass-to-light ratio is found (D'Amico et al. 2002), which possibly indicates the existence of a massive $\mathrm{BH}$ in the centre. The dynamical evolution of GCs depends on the most massive components of the stellar population, i.e. BHs and neutron stars. There are believed to be a population of stellar-mass BHs in addition to the neutron stars, as remnants of massive stellar objects (see e.g. Kulkarni et al. 1993; Sigurdsson \& Hernquist 1993). The stellar-mass BH population and MSP population might not be independent, as both of them sink towards the centre under dynamical friction. In fact, an anti-correlation is found for the population of stellar-mass BHs and the population of MSPs if most of MSPs are formed dynamically, because that BHs, if abundant, will dominate the stellar population in the dense core and therefore reduce the chance of MSP formation (Ye et al. 2019). Observation of MSPs in GCs can put constraints on the stellar-mass BH population, which, together with the observation of existing $\mathrm{BH}$ population, can provide clues to the early dynamical evolution of GCs.

The MSP can lose sufficient amount of energy and consequently be captured by the massive $\mathrm{BH}$ after a relativistic fly-by $\left(r_{\text {min }} \leq 151 M\right.$ for $v_{\infty}=10 \mathrm{~km} / \mathrm{s}$ and $M=10^{3} \mathrm{M}_{\odot}$, see Sec. 4.3 for details), merging into the $\mathrm{BH}$ efficiently, contributing to the growth of a nuclear BH. Even one detection of a pulsar-BH scattering event (or null detection) could 
readily place an lower bound (or upper bound) on the event rate of such a binary. This could provide us clues about the formation and growth history of the nuclear BH. In addition, precision testing of GR can be performed using pulsar systems: e.g. the test of orbital evolution driven by gravitational radiation loss using the binary pulsar PSR B1913+16 (Weisberg \& Taylor 2005), and the test of the strong equivalence principle using a triple binary system (Voisin et al. 2020). The discovery of a pulsar can offer precise measurements of the BH's mass and spin. Further, a pulsar-BH system can serve as a perfect laboratory for a variety of tests of GR, including, for example, Lense-Thirring precession (Wex \& Kopeikin 1999), the no-hair theorem (Liu et al. 2012; Psaltis et al. 2016), and signatures to support alternative gravity theories (Liu et al. 2014). A pulsar can tell the nature of the massive dark object in the core of GCs or Galactic nuclei, whether is it a BH or not (Saxton et al. 2016).

\subsection{Multi-messenger astrophysics}

Currently, radio observation is almost the only channel for identification of $\mathrm{MSPs}^{4}$. Presumably, such hyperbolic systems are present in the core of GCs or nuclear regions of galaxies, and searching for such MSP is made possible by deep radio search targeting at the GCs or nuclear region of nearby galaxies. Searching for such MSPs, however, is limited by several factors, including the complicated orbital behaviours and observational difficulties. In a classical point of view, the motion of the MSP and the radio signal are affected by a variety of effects. In the far field (before and after the scattering), the MSP's motion can be approximated with Newtonian theory. As the MSP moves close to the BH, the velocity increases approximately as $\sqrt{M / r}$, and hence the Doppler shift becomes important. The gravitational redshift factor $(\propto M / r)$ also needs to be taken into consideration. The radio emission from the MSP is also lensed by the gravitational potential of the $\mathrm{BH}$ (and also the surrounding stellar components), casting an additional time delay (i.e. Shapiro delay). A proper treatment of these components will allow us to search for the MSP when it is far away from the nuclear BH. As the MSP becomes more and more relativistic, a full GR treatment is required because of the high sensitivity of pulsar timing. This requires not only the solution to MPD equations, including orbital dynamics and spin dynamics (see e.g. Li et al. 2019), but also the general relativistic radiation transfer of the photons (see e.g. Kimpson et al. 2019). An invalid template will lead to the de-phasing of the template with the signal, and as a consequence, a longer observation time can lead to smaller signal-to-noise ratio (SNR), putting an artificial obstacle against finding such a system. We note that when the gravity field is steep, the time derivative of the observed period derivative can be so large that the signal is no longer periodic (see e.g. Blandford et al. 1987; Foster \& Backer 1990). We will use the word "semiperiodic" to describe such a radio signal.

As a special class of EMRB system, the hyperbolic encounter emits a burst of GWs, and are ideal sources for

4 Out of 127 MSPs listed in http://tinyurl.com/fermipulsars, only 10 were not first identified with radio observations. multi-messenger astrophysics. In fact, GW observations can assist the radio observation by using prior knowledge of the location and parameters of such an system deduced from the GW signal. For example, Kimpson et al. (2020a) studied the GW emission from a bounded EMRB system. For an unbounded system, we can use the Newtonian approximation from Capozziello et al. (2008) to estimate the characteristic strain of the GW:

$h=\frac{2}{R}\left\langle\ddot{Q}_{i j} \ddot{Q}^{i j}\right\rangle^{1 / 2} \approx 4 \sqrt{\frac{2}{3}} \frac{1}{R} \frac{m M}{r_{\min }} \cos ^{2} \frac{\Theta}{2} \sqrt{3 \cos \Theta+4}$

where $\Theta$ is the true anomaly, $Q_{i j}$ is the quadrupole mass tensor and $R$ is the distance between this system and earth. The peak value is reached when $\Theta \rightarrow 0$ :

$$
\begin{aligned}
h_{\text {peak }} & =4 \sqrt{\frac{14}{3}} \frac{1}{R} \frac{m M}{r_{\text {min }}}, \\
& \approx 6.2 \times 10^{-19}\left(\frac{R}{10 \mathrm{kpc}}\right)^{-1}\left(\frac{m}{1.5 \mathrm{M}_{\odot}}\right)^{-1}\left(\frac{r_{\text {min }}}{100 M}\right)^{-1}
\end{aligned}
$$

Note that this peak value of the strain is greater than that of a similar EMRI system on circular orbit with radius $r_{\text {min }}$ by a factor of $\sqrt{7 / 3}$, as the velocity at the periapsis is much larger than that of the circular orbit. The time scale of this GW emission can be characterised by the full-width at half maximum of the GW amplitude. Therefore we have $\Theta \rightarrow$ 1.34:

$$
\begin{aligned}
\tau & =\left.2 \sqrt{\frac{\alpha^{3}}{M}}(e \sinh \epsilon-\epsilon) \approx \frac{4 \sqrt{2} r_{\min }^{3 / 2}}{3 \sqrt{G M}} \frac{(\cos \Theta+2)}{(\cos \Theta+1)} \tan \frac{\Theta}{2}\right|_{\Theta \rightarrow 1.34}, \\
& \approx 13.4 \mathrm{~s} \times\left(\frac{M}{10^{3} \mathrm{M}_{\odot}}\right)\left(\frac{r_{\min }}{100 M}\right)^{3 / 2},
\end{aligned}
$$

where $\epsilon$ is the eccentric anomaly, $\alpha$ is the (positive) semimajor axis of the hyperbola with $\alpha=b / \sqrt{e^{2}-1}$. If an MSP is found on such a hyperbolic orbit with a $\mathrm{BH}$ in the galactic centre or GCs in nearby galaxies, it will be a very luminous GW source in the LISA band. In fact, for appropriate masses of the $\mathrm{BH}\left(10^{4}-10^{5} \mathrm{M}_{\odot}\right)$, this system can be detected up to $10 \mathrm{Mpc}$, and even $\sim 100 \mathrm{Mpc}$ for optimal orbital parameters by LISA (see Sathyaprakash \& Schutz 2009, for the LISA sensitivity curve). Detecting a radio pulsar at such a large distance is certainly very difficult. However, the GW detection can be used as a trigger, and deep targeted search can be performed by SKA or FAST once the GW source is located on the sky map. The GW data, together with the gamma-ray/x-ray data can be used as a prior for searching of such a semi-periodic radio signal. Due to the high accuracy of radio timing, the discovery of such a semi-periodic radio signal will greatly enhance the scientific gain from such a GW event.

\subsection{Fate of the hyperbolic encounter}

Stars with slow speed can be easily captured by the central massive $\mathrm{BH}$ via gravitational radiation. The energy lost by 
the MSP (per unit mass) is (Quinlan \& Shapiro 1989) ${ }^{5}$ :

$$
\Delta E \approx-\frac{85 \pi \mu M^{5 / 2}}{12 \sqrt{2} r_{\min }^{7 / 2}}
$$

The maximum value of $r_{\text {min }}$ that leads to the capture is given by (Quinlan \& Shapiro 1989):

$$
\begin{aligned}
r_{\text {min }, \mathrm{c}} & \approx\left[\frac{85 \sqrt{2} \pi m M^{5 / 2}}{12 v_{\infty}^{2}}\right]^{2 / 7}, \\
& \approx 151 M\left(\frac{\mu}{1.5 \mathrm{M}_{\odot}}\right)^{2 / 7}\left(\frac{M}{10^{3} \mathrm{M}_{\odot}}\right)^{-2 / 7}\left(\frac{v_{\infty}}{10 \mathrm{~km} / \mathrm{s}}\right)^{-4 / 7}
\end{aligned}
$$

which corresponds to a capture impact parameter:

$$
b_{\mathrm{c}} \approx 5 \mathrm{au}\left(\frac{m}{1.5 \mathrm{M}_{\odot}}\right)^{1 / 7}\left(\frac{M}{10^{3} \mathrm{M}_{\odot}}\right)^{6 / 7}\left(\frac{v_{\infty}}{10 \mathrm{~km} / \mathrm{s}}\right)^{-9 / 7} .
$$

This capture impact parameter is comparable to Eq. 18, and is almost directly proportional to the the mass of the central $\mathrm{BH}$. Thus, a more massive $\mathrm{BH}$, which have a larger capture cross-section, will give more stellar encounter events. Even though captured, the energy of these MSPs are still very large $E \approx E_{0}+\Delta E \approx 1$ that they follow orbits that are nearly unbounded with semi-major axis:

$$
a \approx 2 \times 10^{3} \text { au }\left(\frac{\mu}{1.5 \mathrm{M}_{\odot}}\right)^{-1}\left(\frac{M}{10^{3} \mathrm{M}_{\odot}}\right)^{2}\left(\frac{r_{\min }}{100 M}\right)^{2 / 7},
$$

and period

$$
P \approx 3 \times 10^{3} \text { year }\left(\frac{m}{1.5 \mathrm{M}_{\odot}}\right)^{-3 / 2}\left(\frac{M}{10^{3} \mathrm{M}_{\odot}}\right)^{5 / 2}\left(\frac{r_{\min }}{100 M}\right)^{21 / 4}
$$

We note that $2 \times 10^{3}$ au $\approx r_{\text {inf }}$ for a $10^{3} \mathrm{M}_{\odot} \mathrm{BH}^{6}$, and therefore this orbit will likely deviate from Keplerian motion at $r \gg r_{\text {min }}$ due to the gravity of surrounding stellar objects. This deviation will be more stringent for more massive $\mathrm{BH}$. For simplicity, we ignore the effects of surrounding stellar objects for the time being. Then, for a very close fly-by $r_{\text {min }} \leq 25 \mathrm{M}$ around a $10^{3} \mathrm{M}_{\odot} \mathrm{BH}$, the MSP will return within one year and become a periodic GW and radio source. If we allow for a longer observational time of about, say, 20 years, then a close fly-by with $r_{\text {min }} \leq 44 M$ around $10^{3} / \mathrm{M}_{\odot} \mathrm{BH}$ will be seen by the future generation of radio telescopes. Using a Newtonian approximation, the eccentricity of the captured orbit can be deduced from $L_{z}=\sqrt{a M\left(1-e^{2}\right)}$, and the merger time will be about $T \approx 2 P$ with exactly the same dependencies on $m, M$ and $r_{\min }$ (using radiation formula from Peters 1964), meaning that these MSPs will merge into the BH within few orbital periods, regardless of mass of the $\mathrm{BH}$ and periapsis distance. These MSPs (with $r_{\text {min }} \leq r_{\text {min,c }}$ ) are efficient sources for the growth of the $\mathrm{BH}$.

If we take $v_{\infty}$ to be the dispersion velocity satisfying M$\sigma$ relation, with index 5.1 from McConnell et al. (2011) (or

Note that this is the results for a parabolic orbit. A hyperbolic orbit with $v_{\infty}^{2} r_{\min } \ll 1$ in Newtonian approximation yields the same result.

${ }^{6}$ Assuming $\sigma \approx 18 \mathrm{~km} / \mathrm{s}$. The semi-major axis will be much smaller than the influence radius is $\sigma$ is smaller.
4.38 from Zubovas \& King 2019, will give a similar result). then $r_{\text {min, }} \propto M^{-0.4}$. For $M=10^{5} \mathrm{M}_{\odot}$, MSPs with $r_{\text {min }}>24 M$ can remain unbounded, and for $M=4 \times 10^{6} \mathrm{M}_{\odot}$, MSPs with $r_{\text {min }}>6 M$ remain unbounded (or $r_{\text {min }}>4 M$ if we take $v_{\infty} \approx 100 \mathrm{~km} / \mathrm{s}$ ) and will mix with the stellar objects in the core. Unlike a binary BH which can serve as a energy reservoir and give away sufficient energy to the scattered objects to escape the gravity potential (see e.g. Merritt 2013), a single $\mathrm{BH}$ approximately preserves the energy of the scattered particle (if ignoring gravitational radiation). Energy is lost (instead of gained in case of a binary nuclear $\mathrm{BH}$ ) by gravitational radiation, allowing for the core to gradually contract. Therefore, these unbounded pulsars cannot escape the grayity potential of the core, unless if they have already gained sufficient kinetic energy during scattering with nearby stellar objects, before being scattering by the nuclear BH (however, there is no reason to believe that these pulsars with high kinetic energy will fly-by the nuclear BH, due to much smaller scattering cross section). The rarity of MSPs in the outskirt (i.e. beyond half-light radius) of $\mathrm{GCs}^{7}$ suggest that either a binary nuclear $\mathrm{BH}$ phase does not dominate the GC evolution (e.g. the PSR J1911-5958A is believed to be scattered onto the current orbit by a binary muclear $\mathrm{BH}$, see e.g. Colpi et al. 2002), or most pulsars could not gain sufficient kinetic energy during interactions with stellar objects.

\subsection{Conservative post-Minkowskian treatment of hyperbolic encounters}

The self-force is one of the most important issues yet to resolve for the LISA to observe extreme-mass-ratio systems. While most studies have put focus on systems in elliptical orbits, systems in hyperbolic motions are gaining much attention recently (see e.g. Damour 2018; Bjerrum-Bohr et al. 2018; Bern et al. 2019a,b; Damour 2020, etc), due to the non-degenerate feature of the scattering angle. Extending the MPD equations to include the self-force remains a nontrivial question (Kopeikin 2019). In this section, we only estimate the correction to the deflection angle, which are derived using the post-Minkowskian (PM) method. For hyperbolic orbits, the self-force corrections enter the scattering angle at second post-Minkowskian order. The corrections are given by

$$
\chi_{2 \mathrm{PM}}=-\frac{3}{8} \pi\left(5 E_{0}^{2}-1\right)\left(1-\frac{1}{\sqrt{2\left(E_{0}-1\right) v+1}}\right)
$$

(Damour 2018), where $v \equiv m M /(m+M)^{2}$. For an MSP scattered by a $\mathrm{BH}$ of $M=10^{3} \mathrm{M}_{\odot}$ and $v_{\infty} \ll c$, we have

$\chi_{2 \mathrm{PM}} \approx-3.5 \times 10^{-3}\left(\frac{10^{3} \mathrm{M}_{\odot}}{M}\right)\left(\frac{v_{\infty}}{c}\right)^{2} \mathrm{rad}+O\left(v_{\infty}^{3} / c^{3}\right)$,

and is about $-3.9 \times 10^{-12} \mathrm{rad}$ when $v_{\infty}=10 \mathrm{~km} / \mathrm{s}$. The third order post-Minkowskian correction can be found in

7 Among the 136 MSPs detected in 36 Galactic GCs with known offsets, from http://www.naic.edu/ pfreire/GCpsr.html, only 10 are outside the half-light radius. Note that the observation of MSPs is biased against core because of higher luminosity and steeper gravity potential. Further, as some GCs have only few MSPs identified, this statistics is not necessarily reliable. 
(Bern et al. 2019a,b; Bini et al. 2020; Damour 2020)

$$
\begin{aligned}
\chi_{3 \mathrm{PM}}= & -\frac{2 v \sqrt{E_{0}^{2}-1}}{2\left(E_{0}-1\right) v+1}\left[\frac{2}{3} E_{0}\left(14 E_{0}^{2}+25\right)\right. \\
& \left.+\frac{4\left(4 E_{0}^{4}-12 E_{0}^{2}-3\right)}{\sqrt{E_{0}^{2}-1}} \sinh ^{-1} \sqrt{\frac{E_{0}-1}{2}}\right] \\
\approx & -8 v \frac{v_{\infty}}{c}+O\left(v, v_{\infty}^{3} / c^{3}\right) \\
\approx & -1.2 \times 10^{-2}\left(\frac{10^{3} \mathrm{M}_{\odot}}{M}\right)\left(\frac{v_{\infty}}{c}\right) \mathrm{rad} .
\end{aligned}
$$

This correction is about $-4.0 \times 10^{-7} \mathrm{rad}$ when $v_{\infty}=10 \mathrm{~km} / \mathrm{s}$.

Therefore, for the hyperbolic scatterings that we are interested in, the contribution of the self-force is negligible when $v_{\infty}$ is sufficiently small. For the 2PM self-force, the correction is smaller than $10^{-9} \mathrm{rad}$ when $v_{\infty} \leq 100 \mathrm{~km} / \mathrm{s}$. For the 3PM self-force, the correction is of comparable order of $c_{\theta}$ and $c_{\phi}$ for $v_{\infty}=10 \mathrm{~km} / \mathrm{s}$, when $r_{\min } / M>100$, and is only negligible if $r_{\min } / M$ is sufficiently small.

\section{CONCLUSIONS}

It is believed that a large number of MSPs exist in GCs and Galactic centre, where a massive nuclear $\mathrm{BH}$ is believed to reside. The MSPs sink towards the centre under dynamical friction and undergo violent scattering with nearby stars in the dense stellar environment, potentially forming an unbounded system with the nuclear BH. In this work, we investigate the motion of such an MSP on a hyperbolic orbit around a massive $\mathrm{BH}$ in an astrophysical context. Due to the small dispersion velocities $\leq 100 \mathrm{~km} / \mathrm{s}$ in GCs and Galactic centre, the event rate of such scattering is purely dominated by the gravitational focusing: MSPs with smaller $v_{\infty}$ can easily follow a close fly-by with the nuclear $\mathrm{BH}$. In general, the relativistic effects (including spin's effects of the MSP) are the most prominent for slow speed test objects, due to their smaller inertia. While most of the existing studies on the scattering angle (e.g. the studies on conservative self-force contribution, as shown in Sec. 4.4) focus on the high-energy scattering regime, the effects of the relativistic effects might have been underestimated for astrophysical objects.

The orbit of the MSP is calculated by solving the quadratic-in-spin accurate MPD equations. We compare the orbits of spinning MSPs (with dimensionless spin $\hat{s} \approx$ $5.68 \times 10^{-4}$ ) and the orbits of non-spinning pulsars with otherwise same orbital parameters. The spinning and nonspinning pulsars follow different equations of motion (i.e. choice of $\lambda$ and $C_{Q}$ in in Eq. 10), but the orbits they follow are indistinguishable before they fall towards the BH. During the scattering, the spin-orbit and spin-spin couplings allow the scattering angle to vary by a small amount, which we denote $\Delta \phi_{\text {spin }}$ and $\Delta \theta_{\text {spin }}$. We compare this difference in scattering angle $\Delta \phi_{\text {spin }}$ with the analytical formula for equatorial motion calculated by Bini et al. (2017), and show that our results are consistent at linear order when the spin of the MSP is perpendicular to the orbital plane, validating our numerical calculations. We would like to emphasise that the results presented in this work do not include explicit treatment of self-force. As analysed in the Sec. 4.4, the leading order contribution of the self-force leads to negligible modification of the scattering angle for the systems of our interest (i.e. $\left.v_{\infty} \ll c\right)$. However, this contribution does not necessarily converge for higher order self-force effects. For example, the contribution of $\chi_{3} \mathrm{PM}$ is larger than $\chi_{2} \mathrm{PM}$ by a few order of magnitude.

The deflection angle is about $10^{-6}-10^{-3}\left(M / 10^{3} \mathrm{M}_{\odot}\right)$ for a typically close $\left(r_{\min } \leq 200 M\right)$ fly-by, depending on the orientation of the spin axis. Such a deflection angle can lead to a spatial difference of $30 \mathrm{~km}$ when the MSP reaches $r / M=20-20000$, much smaller than the typical influence radius of a $\mathrm{BH}$ with mass between $10^{3}-4 \times 10^{6} \mathrm{M}_{\odot}$. This criterion allows us to ignore the gravitational interaction of the MSP with surrounding stars during the scattering process. When the deviation between geodesic and non-geodesic orbits reaches $30 \mathrm{~km}$, the predicted pulse-arrival-time using different EOMs can reach $\sim 0.1 \mathrm{~ms}$, which is about $1 / 10$ of the period, and definitely within the sensitivity limits of the current pulsar timing of MSPs. Further, we show that an observation of few hours of the MSP (if we observed the MSP's signal both before and after the scattering) can readily tell this differences in EOMs and the effects of spin for most $r_{\text {min }} \leq 200 M$, for a intermediate-mass $\mathrm{BH}$ with $M=10^{3} \mathrm{M}_{\odot}$. $\mathrm{BHs}$ with larger masses require longer observational time $(\propto M)$, but at the same time, has larger scattering cross sections $\left(\propto M^{2}\right)$, which, in a naive approximation, can lead to much larger event rate.

While $1 \mathrm{~ms}$ is about the shortest period among the currently observed MSPs, pulsars with smaller spin can also be used to measure the effects of its spin and therefore test the MPD equations (and perhaps also to set tighter constraints to the equations of state of neutron stars). We have shown that pulsars with period $10 \mathrm{~ms}$ require hours or days of observations (again, if we observed the pulsar's signal both before and after the scattering), to measure a difference in pulse-arrival-time of $0.1 \mathrm{~ms}$. For a 10-hour observation, the effective scattering cross section of a $10 \mathrm{~ms}$ pulsar (to probe a $30 \mathrm{~km}$ difference in position) is about half of that of an MSP with the same $v_{\infty} \ll c$ and $M=10^{3} \mathrm{M}_{\odot}$.

Although no such events have been found so far, it does not imply that the event rate is negligible. The null detection may be an artefact, because existing techniques based on Fourier transformation and phase folding will likely fail for such semi-periodic radio signals. Detection of such a system requires a different set of templates that include GR effects in all aspects. We show in this work that, including the effects of the MSP's spin via the MPD equations is essential for constructing the templates for the search for such a radio signal.

\section{ACKNOWLEDGEMENTS}

KJL is supported by a PhD Scholarship from the Vinson and Cissy Chu Foundation and by a UCL MAPS Dean's Prize. KW thanks the hospitality of the CUHK Department of Physics during his visits. This research has made use of NASA's Astrophysics Data System. 


\section{DATA AVAILABILITY}

The data underlying this article will be shared on reasonable request to the corresponding author.

\section{REFERENCES}

Abbate F., Mastrobuono-Battisti A., Colpi M., Possenti A., Sippel A. C., Dotti M., 2018, MNRAS, 473, 927

Abdo A. A., et al., 2013, ApJS, 208, 17

Aharon D., Perets H. B., 2015, ApJ, 799, 185

Amaro-Seoane P., Gair J. R., Freitag M., Miller M. C., Mandel I., Cutler C. J., Babak S., 2007, Classical and Quantum Gravity, 24, R113

Antonelli A., Kavanagh C., Khalil M., Steinhoff J., Vines J., 2020, Phys. Rev. D, 102, 124024

Arca-Sedda M., Kocsis B., Brandt T. D., 2018, MNRAS, 479, 900

Bagchi M., Lorimer D. R., Chennamangalam J., 2011, MNRAS, 418,477

Bahramian A., Heinke C. O., Sivakoff G. R., Gladstone J. C., 2013, ApJ, 766, 136

Bartels R., Krishnamurthy S., Weniger C., 2016 , Physical Review Letters, 116, 051102

Baumgardt H., Hilker M., 2018, MNRAS, 478, 1520

Bern Z., Cheung C., Roiban R., Shen C.-H., Solon M. P., Zeng M., 2019a, Phys. Rev. Lett., 122, 201603

Bern Z., Cheung C., Roiban R., Shen C.-H., Solon M. P., Zeng M., 2019b, Journal of High Energy Physics, 2019, 206

Bhattacharya D., van den Heuvel E. P. J., 1991, Phys. Rep., 203, 1

Bini D., Geralico A., 2017, General Relativity and Gravitation, 49,84

Bini D., Geralico A., 2018, Physical Review D, 98, 024049

Bini D., de Felice F., Geralico A., Jantzen R. T., 2005, Classical and Quantum Gravity, 22, 2947

Bini D., Geralico A., Vines J., 2017, Phys. Rev. D, 96, 084044

Bini D., Damour T., Geralico A., 2020, Phys. Rev. D, 102, 024061

Bjerrum-Bohr N. E. J., Damgaard P. H., Festuccia G., Planté L., Vanhove P., 2018, Phys. Rev. Lett., 121, 171601

Blandford R. D., Romani R. W., Applegate J. H., 1987, MNRAS, $225,51 \mathrm{P}$

Brandt T. D., Kocsis B., 2015, ApJ, 812, 15

Calore F., Di Mauro M., Donato F., Hessels J. W. T., Weniger C., 2016, ApJ, 827, 143

Capozziello S., de Laurentis M., de Paolis F., Ingrosso G., Nucita A., 2008, Modern Physics Letters A, 23, 99

Chen B., Compère G., Liu Y., Long J., Zhang X., 2019, Classical and Quantum Gravity, 36, 245011 Y

Chennamangalam J., Lorimer D. R., 2014, MNRAS, 440, L86

Colpi M., Possenti A., Gualandris A., 2002, ApJ, 570, L85

Cordes J. M., Chernoff D. F., 1997, ApJ, 482, 971

Cordes J. M., Lazio T. J. W., 2002, arXiv e-prints, pp astro-ph/0207156

Cordes J. M., Shannon R. M., Stinebring D. R., 2016, ApJ, 817,16

D’Amico N., Possenti A., Fici L., Manchester R. N., Lyne A. G., Camilo F., Sarkissian J., 2002, ApJ, 570, L89

Dai S., Johnston S., Kerr M., Camilo F. o., Cameron A., Toomey L., Kumamoto H., 2020, ApJ, 888, L18

Damour T., 2018, Phys. Rev. D, 97, 044038

Damour T., 2020, Phys. Rev. D, 102, 024060

Damour T., Guercilena F., Hinder I., Hopper S., Nagar A., Rezzolla L., 2014, Phys. Rev. D, 89, 081503

De Vittori L., Gopakumar A., Gupta A., Jetzer P., 2014, Phys. Rev. D, 90, 124066

Deriglazov A. A., Ramírez W. G., 2017, International Journal of Modern Physics D, 26, 1750047
Dixon W. G., 1964, Il Nuovo Cimento, 34, 317

Eckner C., et al., 2018, ApJ, 862, 79

Ehlers J., Rudolph E., 1977, General Relativity and Gravitation, 8, 197

Feldmeier A., et al., 2013, A\&A, 554, A63

Ferrarese L., Merritt D., 2000, ApJ, 539, L9

Foster R. S., Backer D. C., 1990, ApJ, 361, 300

Fragione G., Antonini F., Gnedin O. Y., 2018, MNRAS, 475, 5313

Freire P. C. C., et al., 2011, Science, 334, 1107

Freire P. C. C., et al., 2017, MNRAS, 471, 857

Fruchter A. S., Goss W. M., 2000, ApJ, 536, 865

Gair J. R., Babak S., Sesana A., Amaro-Seoane P., Barausse E., Berry C. P. L., Berti E., Sopuerta C., 2017, in Journal of Physics Conference Series. p. 012021 (arXiv: 1704.00009), doi:10.1088/1742-6596/840/1/012021

Gebhardt K., et al., 2000, ApJ, 539, L13

Gerssen J., van der Marel R. P., Gebhardt K., Guhathakurta P., Peterson R. C., Pryor C., 2002, AJ, 124, 3270

Gonzalez M. E., et al., 2011, ApJ, 743, 102

Han W.-B., 2010, Phys. Rev. D, 82, 084013

Han W.-B., Cheng R., 2017, General Relatiyity and Gravitation, 49,48

Hansen R. O., 1972, Phys. Rev. D, 5, 1021

Hartl M. D., 2003a, Phys. Rev. D, 67, 024005

Hartl M. D., 2003b, Phys. Rev. D, 67, 104023

Heinke C. O., Grindlay J. E., Edmonds P. D., Cohn H. N., Lugger P. M., Camilo F., Bogdanov S, Freire P. C., 2005, ApJ, 625,796

Hobbs G., Lorimer D. R., Lyne A. G., Kramer M., 2005, MNRAS, 360,974

Hooper D., $>$ Linden T., 2016, Journal of Cosmology and Astroparticle Physics, 2016, 018

Hooper D., Cholis I., Linden T., Siegal-Gaskins J. M., Slatyer T. R., 2013, Phys. Rev. D, 88, 083009

Hui C. Y., Cheng K. S., Taam R. E., 2010, ApJ, 714, 1149

Hyman S. D., Frail D. A., Deneva J. S., Kassim N. E., McLaughlin M. A., Kooi J. E., Ray P. S., Polisensky E. J., 2019, ApJ, 876,20

Ibata R., et al., 2009, ApJ, 699, L169

Ivanova N., Heinke C. O., Rasio F. A., Belczynski K., Fregeau J. M., 2008, MNRAS, 386, 553

Jiang Y.-F., Greene J. E., Ho L. C., Xiao T., Barth A. J., 2011, ApJ, 742, 68

Kao J. K., Cho H. T., 2005, Physics Letters A, 336, 159

Keresztes Z., Mikóczi B., 2019, arXiv e-prints, p. arXiv:1907.00974

Keresztes Z., Mikoczi B., 2020, Romanian Astronomical Journal, 30,59

Kidder L. E., 1995, Phys. Rev. D, 52, 821

Kimpson T., Wu K., Zane S., 2019, MNRAS, 486, 360

Kimpson T., Wu K., Zane S., 2020a, MNRAS, 495, 600

Kimpson T., Wu K., Zane S., 2020b, MNRAS, 497, 5421

Kizıltan B., Baumgardt H., Loeb A., 2017, Nature, 542, 203

Kopeikin S. M., 2019, Phys. Rev. D, 99, 084008

Kulkarni S. R., Anderson S. B., Prince T. A., Wolszczan A., 1991, Nature, 349, 47

Kulkarni S. R., Hut P., McMillan S., 1993, Nature, 364, 421

Laarakkers W. G., Poisson E., 1999, ApJ, 512, 282

Lattimer J. M., 2012, Annual Review of Nuclear and Particle Science, 62,485

Li K. J., Wu K., Singh D., 2019, MNRAS, 485, 1053

Liu K., Wex N., Kramer M., Cordes J. M., Lazio T. J. W., 2012, ApJ, 747, 1

Liu K., Eatough R. P., Wex N., Kramer M., 2014, MNRAS, 445,3115

Lorimer D. R., 2008, Living Reviews in Relativity, 11

Lützgendorf N., Gebhardt K., Baumgardt H., Noyola E., Neumayer N., Kissler-Patig M., de Zeeuw T., 2015, A\&A, 581, A1 
Lyne A. G., Lorimer D. R., 1994, Nature, 369, 127

Lyne A. G., Brinklow A., Middleditch J., Kulkarni S. R., Backer D. C., 1987, Nature, 328, 399

Lyne A. G., et al., 1998, MNRAS, 295, 743

Macquart J.-P., Kanekar N., 2015, ApJ, 805, 172

Macquart J. P., Kanekar N., Frail D. A., Ransom S. M., 2010, ApJ, 715, 939

Magorrian J., et al., 1998, AJ, 115, 2285

Majár J., Forgács P., Vasúth M., 2010, Phys. Rev. D, 82, 064041

Malov I. F., Baurov Y. A., 2007, Astronomy Reports, 51, 830

Manchester R. N., 2017, Journal of Astrophysics and Astronomy, 38,42

Manchester R. N., Lyne A. G., Robinson C., D'Amico N., Bailes M., Lim J., 1991, Nature, 352, 219

Mathisson M., 1937, Acta Physica Polonica, 6, 163

McConnell N. J., Ma C.-P., Gebhardt K., Wright S. A., Murphy J. D., Lauer T. R., Graham J. R., Richstone D. O., 2011, Nature, 480, 215

Merritt D., 2013, Dynamics and Evolution of Galactic Nuclei. Princeton Series in Astrophysics Vol. 23, Princeton University Press

Mezcua M., 2017, International Journal of Modern Physics D, 26,1730021

Miller M. C., Hamilton D. P., 2002, MNRAS, 330, 232

Moran E. C., Shahinyan K., Sugarman H. R., Vélez D. O., Eracleous M., 2014, AJ, 148, 136

Nguyen D. D., et al., 2017, ApJ, 836, 237

Noyola E., Gebhardt K., Bergmann M., 2008, ApJ, 676, 1008

Özel F., Freire P., 2016, ARA\&A, 54, 401

Papapetrou A., 1951, Proceedings of the Royal Society of London Series A 209, 248

Pennucci T. T., et al., 2015, ApJ, 808, 81

Perera B. B. P., et al., 2017, MNRAS, 468, 2114

Peters P. C., 1964, Physical Review, 136, 1224

Pleunis Z., et al., 2017, ApJ, 846, L19

Psaltis D., Wex N., Kramer M., 2016, ApJ, 818, 121

Quinlan G. D., Shapiro S. L., 1989, ApJ, 343, 725

Rajwade K. M., Lorimer D. R., Anderson L. D., 2017, MNRAS, 471,730

Ransom S. M., Freire P., Stairs I., Hessels J., Lynch R., DeCesar M., 2020, in American Astronomical Society Meeting Abstracts. American Astronomical Society Meeting Abstracts. p. 302.14

Rea N., et al., 2013, ApJ, 775, L34

Reines A. E., Volonteri M., 2015, ApJ, 813, 82

Reines A. E., Greene J. E., Geha M., 2013, ApJ, 775, 116

Remmen G. N., Wu K., 2013, MNRAS, 430, 1940

Romani R. W., Kerr M., Craig H. A., Johnston S., Cognard I., Smith D. A., 2011, ApJ, 738, 114

Rudiger R., 1981, Proceedings of the Royal Society of London Series A 375,185

Rudiger R., 1983, Proceedings of the Royal Society of London Series A, 385,229

Sathyaprakash B. S., Schutz B. F., 2009, Living Reviews in Relativity, 12, 2

Saxton C. J., Younsi Z., Wu K/, 2016, MNRAS, 461, 4295

Semerák O., 1999, MNRAS, 308, 863

She R., Ho L. C.. Feng H., 2017, ApJ, 842, 131

Sigurdsson S, Hernquist L., 1993, Nature, 364, 423

Singh D., 2005, Phys. Rev. D, 72, 084033

Singh D., Wu K., Sarty G. E., 2014, MNRAS, 441, 800

Smith D. A., et al., 2019, ApJ, 871, 78

Srinivasan G., van den Heuvel E. P. J., 1982, A\&A, 108, 143

Steinhoff J., 2011, Annalen der Physik, 523, 296

Suzuki S., Maeda K.-I., 1997, Phys. Rev. D, 55, 4848

Tulczyjew W., 1959, Acta Physica Polonica, 18, 931

Urbanec M., Miller J. C., Stuchlík Z., 2013, MNRAS, 433, 1903

Velandia N., Tejeiro J. M., 2018, MOMENTO, p. 60
Vines J., 2018, Classical and Quantum Gravity, 35, 084002

Vines J., Kunst D., Steinhoff J., Hinderer T., 2016, Phys. Rev. D, 93, 103008

Vines J., Steinhoff J., Buonanno A., 2019, Phys. Rev. D, 99, 064054

Voggel K. T., et al., 2018, ApJ, 858, 20

Voisin G., Cognard I., Freire P. C. C., Wex N., Guillemot L., Desvignes G., Kramer M., Theureau G., 2020, A\&A, 638, A24

Weisberg J. M., Taylor J. H., 2005, in Rasio F. A., Stairs I. H., eds, Astronomical Society of the Pacific Conference Series Vol. 328, Binary Radio Pulsars. p. 25 (arXiv:astro-ph/0407149)

Wex N., Kopeikin S. M., 1999, ApJ, 514, 388

Wharton R. S., Chatterjee S., Cordes J. M., Deneva J. S., Lazio T. J. W., 2012, ApJ, 753, 108

Witzany V., 2019, Phys. Rev. D, 100, 104030

Witzany V., Steinhoff J., Lukes-Gerakopoulos G., 2019, Classical and Quantum Gravity, 36, 075003

Yao J. M., Manchester R. N., Wang N., 2017, ApJ, 835, 29

Ye C. S., Kremer K., Chatterjee S., Rodriguez C. L., Rasio F. A., 2019, ApJ, 877, 122

Yunes N., Buonanno A., Hughes S. A., Pan Y., Barausse E., Miller M. C., Throwe W., 2011, Phys. Rev. D, 83, 044044

Zelenka O., Lukes-Gerakopoulos G., Witzany V., 2019, arXiv eprints, p. arXiv:1903.00360

Zelenka O., Lukes-Gerakopoulos G., Witzany V., Kopáček O., 2020, Phys. Rev. D, 101, 024037

Zubovas K., King A. 2019, General Relativity and Gravitation, 51, 65

de Menezes R., Cafardo F., Nemmen R., 2019, MNRAS, 486, 851

\section{APPENDIX A: LINEAR-IN-SPIN ANALYTIC} FORMULA

We assume that the spinning MSP (i.e. $\lambda=1$ and $C_{Q} \neq 0$ ) and a non-spinning pulsar (i.e. $\lambda=0$ and $C_{Q}=0$ ) follow the same trajectory before the scattering. That is to say, the 4-velocity of a spinning and non-spinning MSP coincide at large $r \geq 10^{4} M$ before the scattering. The 4-velocity of a spinning MSP is given in Eq. (22) of Bini et al. (2017) (we corrected a typo in the equations):

$$
\begin{aligned}
\frac{\Delta}{M^{2}} \frac{d t}{d \tau}= & \hat{E}\left(\frac{r^{2}}{M^{2}}+\hat{a}^{2}\right)+\frac{M}{r}[(2 \hat{a}+3 s) a \hat{E}-(2 \hat{a}+s) \hat{J}] \\
& -\frac{M^{3}}{r^{3}} \hat{a}^{2} s(\hat{J}-\hat{a} \hat{E}) \\
\left(\frac{d r}{d \tau}\right)^{2}= & \hat{E}^{2}-1+\frac{2 M}{r}+\frac{M^{2}}{r^{2}}\left[\hat{a}^{2}\left(\hat{E}^{2}-1\right)-\hat{J}(\hat{J}-2 \hat{E} s)\right] \\
& +\frac{2 M^{3}}{r^{3}}(\hat{J}-\hat{a} \hat{E})(\hat{J}-\hat{a} \hat{E}-3 \hat{E} s) \\
& +\frac{2 M^{5}}{r^{5}} \hat{a} s(\hat{J}-\hat{a} \hat{E})^{2}, \\
\frac{\Delta}{M} \frac{d \phi}{d \tau}= & \hat{J}-\hat{E} s-\frac{2 M}{r}(\hat{J}-\hat{a} \hat{E}-\hat{E} s)-\frac{M^{3}}{r^{3}} \hat{a} s(\hat{J}-\hat{a} \hat{E}),
\end{aligned}
$$

where $\hat{a}$ is the dimensionless spin of the Kerr BH $\hat{a}=a / M$, $\hat{J}$ is the dimensionless angular momentum $\hat{J}=J / M$, and $\hat{E}$ is the energy per unit mass for the pulsar. This 4-velocity equals the 4 -velocity of the non-spinning pulsar whose con- 
stants of motion are:

$$
\begin{aligned}
E_{0} & =\frac{1}{\sqrt{1-v_{\infty}^{2}},} \\
J_{0} & =\frac{\sqrt{\Delta_{\min } r_{\min }\left(\left(E_{0}^{2}-1\right) r_{\min }+2 M\right)}-2 a E_{0} M}{r_{\min }-2 M},
\end{aligned}
$$

where $\Delta_{\min }=a^{2}-2 M r_{\min }+r_{\min }{ }^{2}$. Therefore, we have

$$
\begin{aligned}
\hat{E} & =\frac{E_{0}\left(r_{0}^{3}-a M^{2} s\right)+J_{0} M^{2} s}{r_{0}^{3}-M^{3} s^{2}}, \\
& \approx E_{0}+\frac{M^{2}}{r_{0}^{3}} s\left(J_{0}-a E_{0}\right)+O\left(s^{2}\right), \\
\hat{J} & =\frac{E_{0} M s\left(r_{0}^{3}-a^{2} M\right)+J_{0}\left(a M^{2} s+r_{0}^{3}\right)}{M r_{0}^{3}-M^{4} s^{2}}, \\
& \approx \frac{J_{0}}{M}+s\left(-\frac{a^{2} E_{0} M}{r_{0}^{3}}+\frac{a J_{0} M}{r_{0}^{3}}+E_{0}\right)+O\left(s^{2}\right) .
\end{aligned}
$$

Further, our $\left(e^{\prime}, p^{\prime}\right)$ satisfy

$$
\left.\frac{d r}{d \tau}\right|_{r=M p^{\prime} /\left(1+e^{\prime}\right)}=\left.\frac{d r}{d \tau}\right|_{r=M p^{\prime} /\left(1-e^{\prime}\right)}=0,
$$

and can be found by either solving these two equations with Eq. A1, or equivalently solving Eq. (26-31) in Bini et al. (2017). It is a set of non-linear equations, and the solution does not have a closed form. Therefore, we calculate $\left(e^{\prime}, p^{\prime}\right)$ numerically throughout the paper.

Similarly, the geodetic $e$ and $p$ are defined such that

$\left.\frac{d r}{d \tau}\right|_{r=M p /(1+e)}=\left.\frac{d r}{d \tau}\right|_{r=M p /(1-e)}=0$,

with $\hat{E} \rightarrow E_{0}, \hat{J} \rightarrow J_{0} / M, s \rightarrow 0$ in Eq. A1.

\section{APPENDIX B: ACCURACY OF THE DEFLECTION ANGLE}

For hyperbolic orbits with $v_{\infty} \ll c$, the orbit transits from hyperbolic to parabolic when $v_{\infty}$ is reduced to zero. Therefore, the deflection angle is sensitive to the value of $v_{\infty}$ and hence the value of $E_{0}$. To illustrate this, the variation of geodetic deflection angle $\Delta \phi_{\text {geo }}$ is calculated when $E_{0}$ or $J_{0}$ is perturbed by a small value. We use $\delta_{E_{0}}, \delta_{J_{0}}$ and $\delta_{\Delta \phi_{\text {geo }}}$ to denote the ratio variation of $E_{0}, J_{0}$ and $\Delta \phi_{\text {geo }}$, respectively. As shown in Fig. B1, perturbing $J_{0}$ by $\delta_{J_{0}}$ would only lead to $\delta_{\Delta \phi_{\text {geo }}}$ of comparable order. However, when $E_{0}$ is perturbed, the ratio variation of $\Delta \phi_{\text {geo }}$ is, in general, larger than $\delta_{E_{0}}$. For $v_{\infty} \ll c, \delta_{\Delta \phi_{\text {geo }}}$ is larger than $\delta_{E_{0}}$ by a few order of magnitude, and is approximately inversely proportional to $v_{\infty}$. For the main system $\left(v_{\infty}=10 \mathrm{~km} / \mathrm{s}\right)$ that is considered in this paper, a small variation of $\delta_{E_{0}}=5 \times 10^{-15}$ can lead to a variation of about $10^{-9} \mathrm{rad}$ on $\Delta \phi_{\mathrm{geo}}$. For the same reason, the integration of the EOMs should be performed carefully. A seemingly small variation in the energy can lead to unphysical results in the deflection angle.

This paper has been typeset from a $\mathrm{T}_{\mathrm{E}} \mathrm{X} / \mathrm{LAT} \mathrm{T} \mathrm{X}$ file prepared by the author.
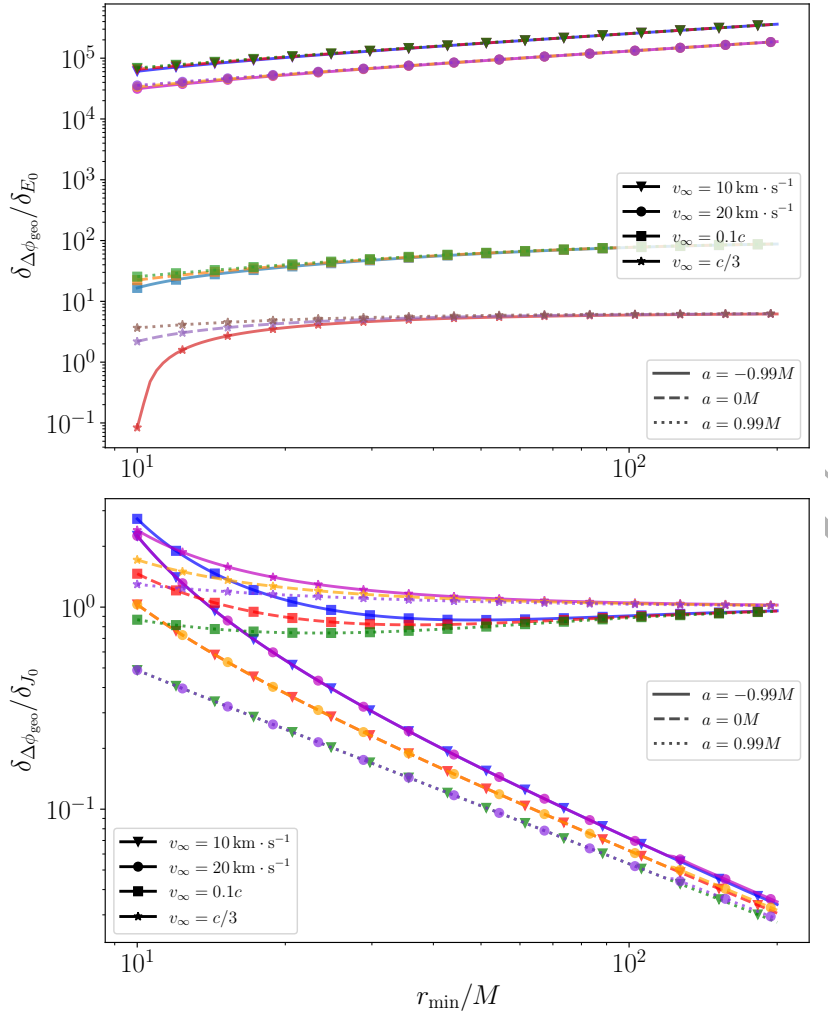

Figure B1. The yariation of the deflection angle $\Delta \phi_{\text {geo }}$ when the initial energy $E_{0}$ or angular momentum $J_{0}$ is perturbed by a small amount. 